\title{
MATHEMATICAL ANALYSIS OF THE STABILIZATION OF LAMELLAR PHASES BY A SHEAR STRESS
}

\author{
V. TORRI ${ }^{1}$
}

\begin{abstract}
We consider a 2D mathematical model describing the motion of a solution of surfactants submitted to a high shear stress in a Couette-Taylor system. We are interested in a stabilization process obtained thanks to the shear. We prove that, if the shear stress is large enough, there exists global in time solution for small initial data and that the solution of the linearized system (controlled by a nonconstant parameter) tends to 0 as $t$ goes to infinity. This explains rigorously some experiments.
\end{abstract}

Mathematics Subject Classification. 35B35, 35Q35, 76E05, 76U05.

Received May 4, 2000. Revised February 16 and August 9, 2001.

\section{INTRODUCTION}

\subsection{Description of the experiment}

Lamellar phases are structures that are created by adding a large quantity of surfactants in water. Molecules of surfactants (like soap) have a particular form: an hydrophile part and an hydrophobe one. When the concentration of surfactants in water is large enough, the molecules organise themselves in order to minimize the interaction with water. Some bilayers of molecules appear in order to form the lamellar phase as showed in figure 1.

When such structures are submitted to a shear in a Couette-Taylor experiment, different cases are observed: if the shear stress is large enough, then these structures are stable. Otherwise some instabilities appear and lead to the formation of more complicated structures: the layers break themselves and are reorganised in concentric spheres which size is of order of the micrometer and that are called spherulits or "onions" (they have the structure of an onion!) ( $c f .[5,6])$. Recently, a mathematical model has been written in order to try to explain this instability (see [3]). It relies on the fact that the Couette-Taylor system ( $c f$. Fig. 2: two coaxial cylinders: the solution being between the cylinders) is never perfect and that the cylinders are never really coaxial. Even if the strain is not large (1\%), this can induce huge forces acting on the layers since the thickness of the layers is that of two molecules. The numerical results of [3] show that this is in fact the right explanation for this instability and correspond to the experiments. It is also observed in the experiments that at high shear, the instability does not develop and that the small scales are always stable.

The aim of this paper is to prove these facts from the model. We show that the small scales are always linearly stable (implied by Prop. 3.6) and that when the shear stress is large enough, then the lamellar phase is linearly and nonlinearly stable (cf. Th. 3.1 and Th. 4.1).

Keywords and phrases: Stabilization, shear stress, Couette system, global solution.

1 Mathématiques Appliquées de Bordeaux, Université de Bordeaux 1 et UMR 5466 du CNRS, 351 cours de la Libération,

33405 Talence Cedex, France; e-mail: torri@math.u-bordeaux.fr 


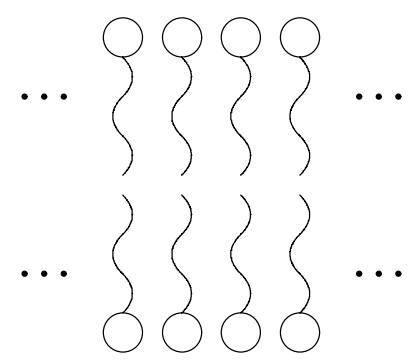

(a) Detail of a layer

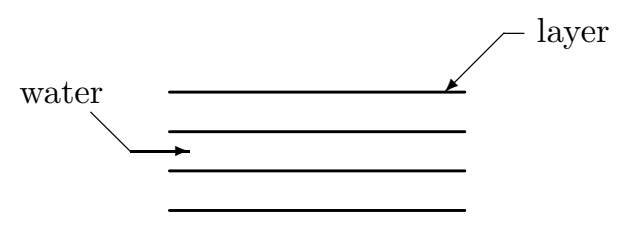

(b) Lamellar phase

Figure 1. Structure of lamellar phases.

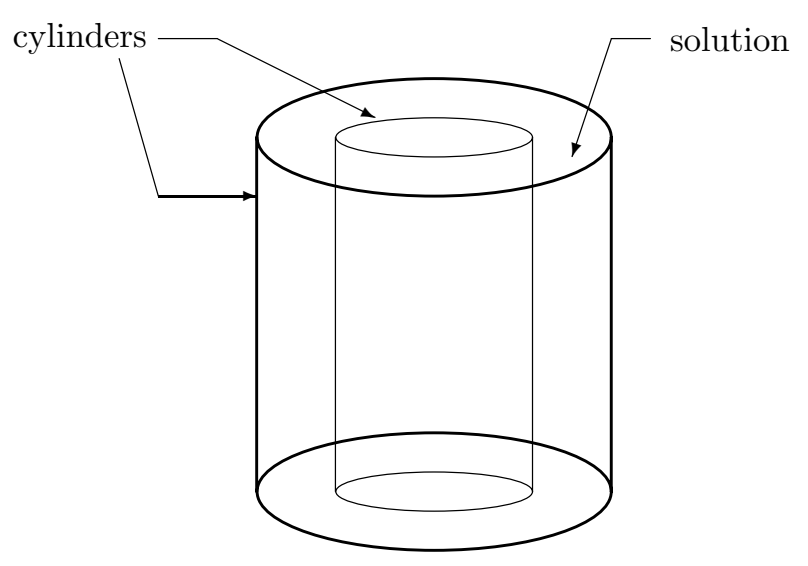

Figure 2. Couette-Taylor system.

\subsection{Description of the model}

We briefly give the physical model that decribes the facts presented in the previous section.

As we neglect the boundary effects, we consider an horizontal section of the Couette-Taylor system. In Figure $3 \mathrm{a}$ is represented the coordinate system. The deplacement field $h$ and the velocity field $(u, v)$ are given by Figure 3b (the grey rectangles are the inner and the outer cylinders of the Couette-Taylor system, the black lines are the lamellar phase at equilibrium and one layer is represented around its equilibrium position).

The Navier-Stokes equations link $u, v$ and $h$ and the system is closed by the motion of a layer:

$$
\begin{aligned}
\rho\left(\partial_{t} U+(U . \nabla) U\right) & =-\nabla p+F_{\text {visq }}+G_{z}(h) \\
\nabla . U & =0 \\
\partial_{t}+(U . \nabla) h & =v+\lambda_{p} G_{z}(h),
\end{aligned}
$$




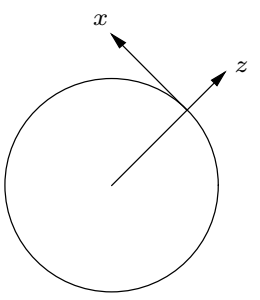

(a) Coordinate system

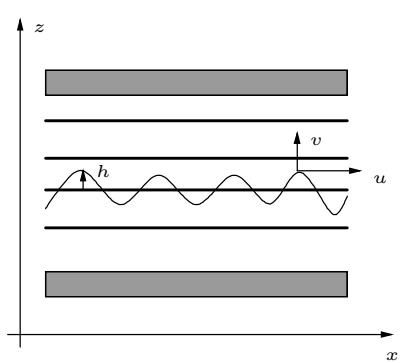

(b) Velocity and displacement field

Figure 3

where $F_{\text {visq }}$ is a viscous force, $\lambda_{p}$ a small constant and $G_{z}$ the force describing the interaction between the layers and the water:

$$
G_{z}(h)=-\frac{\partial F}{\partial h}
$$

where $F(h)=\frac{B}{2}\left(\partial_{z} h-\frac{1}{2}\left(\partial_{x} h\right)^{2}\right)^{2}+\frac{1}{2} K\left(\partial_{x}^{2} h\right)^{2} . B$ is the compression modulus (that is how the layers react when they are only compressed) and $K$ the curvature modulus (that is how the layers react when they are only bent).

We consider the system around a steady solution of these equations. After some hypothesis, one of the two components of the velocity field can be deduced from the other. Then the system is reduced to a system of two equations with the unkowns $v(t, x, z)$ (the radial velocity) and $h(t, x, z)$ (the relative displacement of a layer with respect to the state of the rest). Finally, the system is written in a nondimensionnal form (cf. [3] for a detailed obtention of these equations):

$$
\begin{aligned}
\partial_{t} v+z \partial_{x} v= & \lambda \alpha \partial_{x}^{2} v+\lambda \beta \partial_{z}^{2} v+\lambda^{2} \gamma_{1} \partial_{z}^{2} h-\lambda^{2} \delta_{1} \partial_{x}^{2} h-\lambda^{2} \epsilon_{1} \partial_{x}^{4} h \\
& +\mu \lambda^{2} \theta_{1} \partial_{x}\left(\partial_{x} h \partial_{z} h\right)+\mu \lambda^{2} \theta_{2} \partial_{z}\left(\partial_{x} h\right)^{2}+\nu^{2} \lambda^{3} \theta_{3} \partial_{x}\left(\partial_{x} h\right)^{3} \\
\partial_{t} h+z \partial_{x} h= & v+\lambda \gamma_{2} \partial_{z}^{2} h-\lambda \delta_{2} \partial_{x}^{2} h-\lambda \epsilon_{2} \partial_{x}^{4} h
\end{aligned}
$$

We note by $\lambda^{-1}$ the velocity of the outer cylinder. It is suppose to be great, so $\lambda$ is small. The physical parameters $\alpha, \beta, \gamma_{i}, \delta_{i}, \epsilon_{i}(i=1,2), \theta_{i}(i=1,2,3), \mu$ and $\nu$ are positive and depend on physical constants and datas (such as density, compression modulus...) ( $c f .[3]$ ). The last two parameters depend on the velocity of the shear.

We conclude this description by the boundary conditions:

- $v$ and $h$ are periodic in the $x$ direction (as the solution is between two cylinders);

- They vanish at $z=0,1$.

The main question concerning this system is the stability of the zero solution, that is, do we have global existence of a bounded solution for small data? The first question is therefore: is zero linearly stable? By changing $h$ in $\tilde{h}=\frac{1}{\lambda} h$ and $t$ by $\lambda t$, the linearized version (around zero) of this system is:

$$
\begin{aligned}
\partial_{t} v+\frac{1}{\lambda} z \partial_{x} v & =\alpha \partial_{x}^{2} v+\beta \partial_{z}^{2} v+\gamma_{1} \partial_{z}^{2} h-\delta_{1} \partial_{x}^{2} h-\epsilon_{1} \partial_{x}^{4} h \\
\partial_{t} h+\frac{1}{\lambda} z \partial_{x} h & =v+\gamma_{2} \partial_{z}^{2} h-\delta_{2} \partial_{x}^{2} h-\epsilon_{2} \partial_{x}^{4} h .
\end{aligned}
$$


Recall that $\frac{1}{\lambda}$ corresponds to the angular velocity of the outer cylinder of the Couette system. If one takes $\frac{1}{\lambda}=0$, expanding $h$ and $v$ in Fourier series with respect to $x$ and $z$ gives $(k$ and $l$ are the Fourier variables corresponding respectively to $x$ and $z$ ):

$$
\begin{aligned}
& \partial_{t} v=-\left(\alpha k^{2}+\beta l^{2}\right) v+\left(-\gamma_{1} l^{2}+\delta_{1} k^{2}-\epsilon_{1} k^{4}\right) h \\
& \partial_{t} h=v+\left(-\gamma_{2} l^{2}+\delta_{2} k^{2}-\epsilon_{2} k^{4}\right) h .
\end{aligned}
$$

The determinant of the matrix of the right-hand-side is equal to

$$
\left(\alpha k^{2}+\beta l^{2}\right)\left(\gamma_{2} l^{2}-\delta_{2} k^{2}+\epsilon_{2} k^{4}\right)+\left(\gamma_{1} l^{2}-\delta_{1} k^{2}+\epsilon_{1} k^{4}\right)
$$

which can be negative (for small $k$ and small $l$ ). Hence, the solution can be unbounded as $t$ goes to infinity.

The aim of this paper is to prove that if $\lambda$ is small enough, then these small frequencies are stable. Recent results $(c f .[2,7,8])$ show that skew-symmetric operators with large coefficients have some smoothing effects in nonlinear problems. Here, since the operator $\frac{1}{\lambda} z \partial_{x}$ does not have constant coefficient, this smoothing appears in the linear case, see Section 2.3 for a simple example.

\section{Statement of the main Results}

\subsection{Functionnal spaces and notations}

The system of coordinates is $(x, z) \in \Omega=(-1,1) \times(0,1)$. We will consider periodic functions in the $x$ direction which vanish at $z=0,1$.

Let us define $B=\mathbb{R} \times(0,1)$. Let

$$
\begin{gathered}
\mathrm{L}(\Omega)=\left\{f \in \mathrm{L}_{\text {loc }}^{2}(\bar{B}), 2 \text {-periodic in the } x \text { direction a.e. in } z\right\} \\
\mathrm{H}(\Omega)=\left\{f \in \mathrm{L}(\Omega), f_{\mid \Omega} \in \mathrm{H}^{1}(\Omega), 2 \text {-periodic in } x, f(x, 0)=f(x, 1)=0\right\}
\end{gathered}
$$

and

$$
\mathrm{K}(\Omega)=\left\{f \in \mathrm{H}(\Omega), \partial_{x}^{2} f \in \mathrm{L}^{2}(\Omega)\right\}
$$

We will denote $|u|_{p}=\left(\int_{\Omega}|u|^{p}\right)^{\frac{1}{p}}$ and $\mathrm{H}^{s}$ the usual Sobolev spaces. We note by $C_{p}$ the Poincaré's constant, which verifies

$$
|h|_{2} \leqslant C_{p}\left|\partial_{x} h\right|_{2}
$$

for $h \in \mathrm{H}(\Omega)$.

\subsection{Statement of the main results}

Let us recall that the system reads as follows:

$$
\begin{aligned}
\partial_{t} v+z \partial_{x} v= & \lambda \alpha \partial_{x}^{2} v+\lambda \beta \partial_{z}^{2} v+\lambda^{2} \gamma_{1} \partial_{z}^{2} h-\lambda^{2} \delta_{1} \partial_{x}^{2} h-\lambda^{2} \epsilon_{1} \partial_{x}^{4} h \\
& +\mu \lambda^{2} \theta_{1} \partial_{x}\left(\partial_{x} h \partial_{z} h\right)+\mu \lambda^{2} \theta_{2} \partial_{z}\left(\partial_{x} h\right)^{2}+\nu^{2} \lambda^{3} \theta_{3} \partial_{x}\left(\partial_{x} h\right)^{3} \\
\partial_{t} h+z \partial_{x} h= & v+\lambda \gamma_{2} \partial_{z}^{2} h-\lambda \delta_{2} \partial_{x}^{2} h-\lambda \epsilon_{2} \partial_{x}^{4} h
\end{aligned}
$$


(cf. Sect. 1). By changing $h$ in $\tilde{h}=\lambda h$ and scaling in time (replacing $t$ by $\lambda t$ ), we obtain:

$$
\begin{aligned}
\partial_{t} v+\frac{1}{\lambda} z \partial_{x} v= & \alpha \partial_{x}^{2} v+\beta \partial_{z}^{2} v+\gamma_{1} \partial_{z}^{2} h-\delta_{1} \partial_{x}^{2} h-\epsilon_{1} \partial_{x}^{4} h \\
& +\frac{\mu}{\lambda} \theta_{1} \partial_{x}\left(\partial_{x} h \partial_{z} h\right)+\frac{\mu}{\lambda} \theta_{2} \partial_{z}\left(\partial_{x} h\right)^{2}+\frac{\nu^{2}}{\lambda} \theta_{3} \partial_{x}\left(\partial_{x} h\right)^{3} \\
\partial_{t} h+\frac{1}{\lambda} z \partial_{x} h= & v+\gamma_{2} \partial_{z}^{2} h-\delta_{2} \partial_{x}^{2} h-\epsilon_{2} \partial_{x}^{4} h,
\end{aligned}
$$

and $(x, z) \in \Omega$.

For sake of simplicity, we omit the coefficients of the nonlinear terms $\left(\theta_{1}, \theta_{2}\right.$ and $\left.\theta_{3}\right)$ and those of the viscous terms $\left(\alpha, \beta, \gamma_{1}, \gamma_{2}, \epsilon_{1}\right.$ and $\left.\epsilon_{2}\right)$. They do not have an important role in the proofs and give complicated formulae. We just keep $\delta_{1}$ and $\delta_{2}$. Here is the system we will study:

$$
\begin{aligned}
\partial_{t} v+\frac{1}{\lambda} z \partial_{x} v= & \Delta v+\partial_{z}^{2} h-\delta_{1} \partial_{x}^{2} h-\partial_{x}^{4} h \\
& +\frac{\mu}{\lambda} \partial_{x}\left(\partial_{x} h \partial_{z} h\right)+\frac{\mu}{\lambda} \partial_{z}\left(\partial_{x} h\right)^{2}+\frac{\nu^{2}}{\lambda} \partial_{x}\left(\partial_{x} h\right)^{3} \\
\partial_{t} h+\frac{1}{\lambda} z \partial_{x} h= & v+\partial_{z}^{2} h-\delta_{2} \partial_{x}^{2} h-\partial_{x}^{4} h .
\end{aligned}
$$

The main result of this paper is the stabilization of the lamellar phase at high shear, i.e. the global existence of a solution of $(3,4)$ (therefore of $(1,2)$ ) for small $\lambda$ and small initial data and convergence to 0 of the solution of the linearised system as $t$ goes to infinity. More precisely, we have:

Theorem 2.1. There exists $\lambda_{0}>0$ such that for all $\lambda$ in $\left(0, \lambda_{0}\right)$, for all $\left(v_{0}, h_{0}\right)$ in $\mathrm{L}(\Omega) \times \mathrm{H}(\Omega)$, the linearised system of $(3,4)$ has a unique solution $(v, h)$ defined on $\mathbb{R}_{+}$and which satisfies $v(0)=v_{0}, h(0)=h_{0}$ and

$$
(v, h)(t) \underset{t \rightarrow+\infty}{\longrightarrow} 0 \text { in } \mathrm{L}(\Omega) \times \mathrm{K}(\Omega) .
$$

This stabilization result is obtained thanks to energy estimates.

Theorem 2.2. There exists $0<\lambda_{0}<1$ and $M>0$ such that, for all $0<\lambda<\lambda_{0}, v_{0}$ in $\mathrm{L}(\Omega)$, $h_{0}$ in $\mathrm{K}(\Omega)$ such that $\left(\left|v_{0}\right|_{2}^{2}+\left|h_{0}\right|_{2}^{2}+\left|\partial_{x}^{2} h_{0}\right|_{2}^{2}+\lambda\left|\partial_{z} h_{0}\right|_{2}^{2}\right)<M$, for all $\mu<\lambda^{\frac{5}{2}}, \nu<\lambda^{\frac{5}{4}}$, there exists $v, h: \mathbb{R}_{+} \times \Omega \rightarrow \mathbb{R}$ such that

- $v \in \mathrm{L}^{\infty}\left(\mathbb{R}_{+} ; \mathrm{L}(\Omega)\right) \cap \mathrm{L}^{2}\left(\mathbb{R}_{+} ; \mathrm{H}(\Omega)\right) \cap \mathrm{C}^{0}\left(\mathbb{R}_{+} ; \mathrm{L}^{2}(\Omega)\right)$;

- $h \in \mathrm{L}^{\infty}\left(\mathbb{R}_{+} ; \mathrm{K}(\Omega)\right) \cap \mathrm{L}^{2}\left(\mathbb{R}_{+} ; \mathrm{H}(\Omega) \cap \mathrm{H}^{2}(\Omega)\right) \cap \mathrm{C}^{0}\left(\mathbb{R}_{+} ; \mathrm{L}^{2}(\Omega)\right)$;

- $\partial_{x}^{2} \partial_{z} h \in \mathrm{L}^{2}\left(\mathbb{R}_{+} \times \Omega\right)$;

- $\partial_{x}^{4} h \in \mathrm{L}^{2}\left(\mathbb{R}_{+} \times \Omega\right)$;

solutions of (3) and (4) such that $v(0)=v_{0}$ and $h(0)=h_{0}$.

Remark 2.3. It is not easy to estimate $\lambda_{0}$ in terms of the physical parameters. However, in [3], the numerical value of $\lambda_{0}$ corresponds to that of the experiment.

We also give a local existence theorem whatever the velocity of the shear and the initial data are:

Theorem 2.4. There exists $T_{e}>0$ such that, for all $v_{0}$ in $\mathrm{L}(\Omega)$ and $h_{0}$ in $\mathrm{K}(\Omega)$, there exists $v, h:\left[0, T_{e}[\times \Omega \rightarrow\right.$ $\mathbb{R}$ such that for all $T$ in $] 0, T_{e}[$,

- $v \in \mathrm{L}^{\infty}(0, T ; \mathrm{L}(\Omega)) \cap \mathrm{L}^{2}(0, T ; \mathrm{H}(\Omega)) \cap \mathrm{C}^{0}\left([0, T] ; \mathrm{L}^{2}(\Omega)\right)$;

- $h \in \mathrm{L}^{\infty}(0, T ; \mathrm{K}(\Omega)) \cap \mathrm{L}^{2}\left(0, T ; \mathrm{H}(\Omega) \cap \mathrm{H}^{2}(\Omega)\right) \cap \mathrm{C}^{0}\left([0, T] ; \mathrm{L}^{2}(\Omega)\right)$; 
- $\partial_{x}^{2} \partial_{z} h \in \mathrm{L}^{2}\left(\mathbb{R}_{+} \times \Omega\right)$;

- $\partial_{x}^{4} h \in \mathrm{L}^{2}\left(\mathbb{R}_{+} \times \Omega\right)$;

solutions of (3) and (4) such that $v(0)=v_{0}$ and $h(0)=h_{0}$.

This paper is organised as follows:

- the Section 2.3 gives a short example of an equation which shows the main ideas used to prove Theorem 2.2;

- Section 3 is devoted to energy estimates for the linear case and to the proof of Theorem 2.1. As the system is periodic in the $x$ direction, we compute the fourier coefficients of $v$ and $h$. We deal with both local and global in time cases;

- Section 4 is devoted to a priori estimates of the nonlinear terms that appear in equation (3). Here again we give estimates for the global and the local in time cases;

- Sections 5 and 6 are devoted respectively to the proof of Theorem 2.2 and Theorem 2.4.

\subsection{Example}

One of the main difficulties of this paper will be reviewed on the following example: find a bounded solution of the equation

$$
\partial_{t} u+\frac{1}{\lambda} z \partial_{x} u=\Delta u+\alpha^{2} u
$$

provided $\lambda$ small, where $\alpha$ and $\lambda$ are two positive constants, $u: \Omega \rightarrow \mathbb{R} 2$-periodic in $x$ and vanishing at $z=0,1$, with a mean value with respect to $x$ equal to zero. The method used here is the Galerkin method which requires a priori estimates.

In this section, if $u$ is a solution of (5), then we consider $N(t)=|u|_{2}^{2}+\left|\partial_{x} u\right|_{2}^{2}+\lambda\left|\partial_{z} u\right|_{2}^{2}$ and $\tilde{N}(t)=$ $N(t)-C \sqrt{\lambda} \int_{\Omega} \partial_{x} u \partial_{z} u$, where $C$ is a constant wich will be precised later. Then we have the following result:

Theorem 2.5. There exists $\lambda_{0}>0$ such that, for all $\lambda$ in $\left(0, \lambda_{0}\right)$, for all $u_{0}$ in $\mathrm{H}^{1}(\Omega)$ with a mean value with respect to $x$ equal to zero, there exists $u$ in $\mathrm{L}^{\infty}\left(\mathbb{R}_{+} ; \mathrm{H}^{1}(\Omega)\right) \cap \mathrm{C}^{0}\left(\mathbb{R}_{+} ; \mathrm{H}^{1}(\Omega)\right)$ such that $u$ verifies (5) and $u(0)=u_{0}$. Moreover,

$$
|u(t)|_{H^{1}} \underset{t \rightarrow+\infty}{\longrightarrow} 0 .
$$

Proof. This result is a straightforward corollary of the following lemma:

Lemma 2.6. Let $C<\frac{1}{\sqrt{2}}$. There exists $A>0$ and $\lambda_{0}>0$ such that, for all solution $u$ of (5), for all $\lambda$ in $\left(0, \lambda_{0}\right)$ and for all $t$ in $[0, T)$,

$$
\tilde{N}^{\prime}(t)+\frac{1}{2}\left|\partial_{z} u\right|_{2}^{2}+\left|\partial_{x} \partial_{z} u\right|_{2}^{2}+\lambda\left|\partial_{z}^{2} u\right|_{2}^{2}+\frac{C}{2 \sqrt{\lambda}}\left|\partial_{x} u\right|_{2}^{2} \leqslant-A N(t) .
$$

Proof. We first perform a Fourier transform with respect to $x, \hat{u}(k, z)=\frac{1}{2} \int_{-1}^{1} \exp (-i k \pi s) u(s, z) \mathrm{d} s$, still denoted by $u$. Then we get the following equation:

$$
\partial_{t} u+\frac{i k}{\lambda} z u=-k^{2} u+\partial_{z}^{2} u+\alpha^{2} u
$$

Let $\Omega_{z}=(0,1)$ and $N_{k}(t)=|u|_{2}^{2}+k^{2}|u|_{2}^{2}+\lambda\left|\partial_{z} u\right|_{2}^{2}$ and $\tilde{N}_{k}(t)=N_{k}(t)-C k \sqrt{\lambda} \operatorname{Im} \int_{\Omega_{z}} u \partial_{z} \bar{u}$. We compute $\tilde{N}_{k}^{\prime}(t)$ :

$$
\frac{1}{2} \partial_{t}|u|_{2}^{2}=\left(\alpha^{2}-k^{2}\right)|u|_{2}^{2}-\left|\partial_{z} u\right|_{2}^{2}
$$

as the resulting imaginary term is equal to zero. In the same way,

$$
\frac{1}{2} \partial_{t}\left|\partial_{z} u\right|_{2}^{2}=\frac{k}{\lambda} \operatorname{Im} \int_{\Omega_{z}} u \partial_{z} \bar{u}+\left(\alpha^{2}-k^{2}\right)\left|\partial_{z} u\right|_{2}^{2}-\left|\partial_{z}^{2} u\right|_{2}^{2}
$$


Finally,

$$
\begin{aligned}
\frac{1}{2} \partial_{t} \operatorname{Im} \int_{\Omega_{z}} \partial_{z} \bar{u} u & =-\frac{k}{\lambda} \operatorname{Re} \int_{\Omega_{z}} z u \partial_{z} \bar{u}+\left(\alpha^{2}-k^{2}\right) \operatorname{Im} \int_{\Omega_{z}} u \partial_{z} \bar{u}+\operatorname{Im} \int_{\Omega_{z}} \partial_{z}^{2} u \partial_{z} \bar{u} \\
& =\frac{k}{2 \lambda}|u|_{2}^{2}+\left(\alpha^{2}-k^{2}\right) \operatorname{Im} \int_{\Omega_{z}} u \partial_{z} \bar{u}+\operatorname{Im} \int_{\Omega_{z}} \partial_{z}^{2} u \partial_{z} \bar{u}
\end{aligned}
$$

by integrating by parts the first term of the right member. This term is the one which will be significant in the proof (thanks to the $\frac{1}{\lambda}|u|_{2}$ term). We therefore obtain

$$
\begin{aligned}
\frac{1}{2} \tilde{N}_{k}^{\prime}(t)= & \left(\alpha^{2}-k^{2}\right)|u|_{2}^{2}-\left|\partial_{z} u\right|_{2}^{2}+k^{2}\left(\alpha^{2}-k^{2}\right)|u|_{2}^{2}-k^{2}\left|\partial_{z} u\right|_{2}^{2} \\
& +k \operatorname{Im} \int_{\Omega_{z}} u \partial_{z} \bar{u}+\lambda\left(\alpha^{2}-k^{2}\right)\left|\partial_{z} u\right|_{2}^{2}-\lambda\left|\partial_{z}^{2} u\right|_{2}^{2} \\
& -\frac{C k^{2}}{2 \sqrt{\lambda}}|u|_{2}^{2}-C k \sqrt{\lambda}\left(\alpha^{2}-k^{2}\right) \operatorname{Im} \int_{\Omega_{z}} u \partial_{z} \bar{u}-C k \sqrt{\lambda} \operatorname{Im} \int_{\Omega_{z}} \partial_{z}^{2} u \partial_{z} \bar{u} \\
\leqslant & \left(\left(\alpha^{2}-\frac{k^{2}}{2}\right)+\left(\frac{C^{2}}{2}-1\right) k^{4}+k^{2} \alpha^{2}\left(1-C^{2}\right)+\frac{1}{2} C^{2} \alpha^{4}\right)|u|_{2}^{2} \\
& -\frac{1}{2}\left|\partial_{z} u\right|_{2}^{2}+\lambda\left(\alpha^{2}-\frac{k^{2}}{2}\right)\left|\partial_{z} u\right|_{2}^{2}-\frac{\lambda}{2}\left|\partial_{z}^{2} u\right|_{2}^{2}-\frac{C k^{2}}{2 \sqrt{\lambda}}|u|_{2} .
\end{aligned}
$$

We now deal separately with high frequencies ( $k$ large) and small ones ( $k$ small).

- Study for high frequencies: since $C<\frac{1}{\sqrt{2}}<1$, there exists $K>0$ (depending only on $\alpha$ and $C$ ) so that, for all $k>K$,

$$
\tilde{N}_{k}^{\prime}(t) \leqslant-\frac{k^{2}}{2} N_{k}(t)-\left|\partial_{z} u\right|_{2}^{2}-k^{2}\left|\partial_{z} u\right|_{2}-\lambda\left|\partial_{z}^{2} u\right|_{2}^{2}-\frac{C k^{2}}{2 \sqrt{\lambda}}|u|_{2}^{2} .
$$

- Study for small frequencies: since the mean value of $u$ is equal to zero (it is easy to show, by computing $|u|_{2}$ and by using Gronwall's lemma, that the mean value with respect to $x$ remains equal to 0 ), we only need to study $\tilde{N}_{k}^{\prime}(t)$ for $k$ between 1 and $K$. By $(7)$,

$$
\begin{aligned}
\tilde{N}_{k}(t) \leqslant & -k^{2} N_{k}(t)+\left(2 \lambda \alpha^{2}-1\right)\left|\partial_{z} u\right|_{2}^{2}-k^{2}\left|\partial_{z} u\right|_{2}^{2}-\lambda\left|\partial_{z}^{2} u\right|_{2}^{2} \\
& +\left(2 \alpha^{2}-2 k^{2}\left(2 C^{2} \alpha^{2}-1\right)+C^{2} \alpha^{4}-\frac{C k^{2}}{\sqrt{\lambda}}\right)|u|_{2}
\end{aligned}
$$

So for small $\lambda$, for all $k$ between 1 and $K$, we have

$$
\tilde{N}_{k}^{\prime}(t) \leqslant-N_{k}(t)-\frac{1}{2}\left|\partial_{z} u\right|_{2}^{2}-k^{2}\left|\partial_{z} u\right|_{2}^{2}-\lambda\left|\partial_{z}^{2} u\right|_{2}^{2}-\frac{C k^{2}}{2 \sqrt{\lambda}}|u|_{2}
$$

Hence, using (8) and (9), we have, for all $k>0$

$$
\tilde{N}_{k}(t) \leqslant-A N_{k}(t)-\frac{1}{2}\left|\partial_{z} u\right|_{2}^{2}-k^{2}\left|\partial_{z} u\right|_{2}^{2}-\lambda\left|\partial_{z}^{2} u\right|_{2}^{2}-\frac{C k^{2}}{2 \sqrt{\lambda}}|u|_{2}
$$

where $A=\min \left(1, \frac{K^{2}}{2}\right)$. We have the same result for $k<0$ by taking the conjugate expression of (6). Then the Parseval-Bessel's identity finishes the proof of the lemma. 
To conclude the proof of Theorem 2.5, it suffices to show that $\tilde{N}(t)$ and $N(t)$ are equivalent, which is obvious if $C$ is sufficiently small (possible as the lemma is valid since $C<\frac{1}{\sqrt{2}}$ ), and to use a Galerkin method, which is quite easy to apply as the equation is linear.

Remark 2.7. This stabilization process (obtained thanks to the linear term $\frac{z}{\lambda} \partial_{x} u$ ) is the one that will be used in the physical case.

\section{LinEAR CASE}

In this section, we study the linearized system of $(3,4)$, i.e.

$$
\begin{aligned}
\partial_{t} v+\frac{1}{\lambda} z \partial_{x} v & =\Delta v+\partial_{z}^{2} h-\delta_{1} \partial_{x}^{2} h-\partial_{x}^{4} h \\
\partial_{t} h+\frac{1}{\lambda} z \partial_{x} h & =v+\partial_{z}^{2} h-\delta_{2} \partial_{x}^{2} h-\partial_{x}^{4} h \\
v(0) & =v_{0} \\
h(0) & =h_{0} .
\end{aligned}
$$

We will show local existence of solutions of such systems and linear stability when $\lambda$ is sufficently small. As the arguments are based on the Galerkin method, we will derive energy estimates envolving quantities like $|v|_{2}^{2}$, $|h|_{2}^{2},\left|\partial_{x}^{2} h\right|_{2}^{2}$ and $\lambda\left|\partial_{z} h\right|_{2}^{2}$. Hence the two following results hold:

Theorem 3.1. Let $\left(v_{0}, h_{0}\right)$ in $\mathrm{L}(\Omega) \times \mathrm{H}(\Omega)$. There exists a unique solution $(v, h)$ to (10-13) such that $(v, h)$ in $\mathrm{C}^{0}\left(\mathbb{R}_{+} ; \mathrm{L}(\Omega) \times \mathrm{K}(\Omega)\right)$ and $v \in \mathrm{L}_{\text {loc }}^{2}\left(\mathbb{R}_{+} ; \mathrm{H}^{1}\right), \partial_{x}^{2} h, \partial_{z} h \in \mathrm{L}_{\text {loc }}^{2}\left(\mathbb{R}_{+} ; \mathrm{L}^{2}(\Omega)\right)$.

Theorem 3.2. There exists $\lambda_{0}>0$ such that for all $\lambda$ in $\left(0, \lambda_{0}\right)$, for all $\left(v_{0}, h_{0}\right)$ in $\mathrm{L}(\Omega) \times \mathrm{H}(\Omega)$, the solution $(v, h)$ given by Theorem 3.1 satisfies

$$
(v, h)(t) \underset{t \rightarrow+\infty}{\longrightarrow} 0 \text { in } \mathrm{L}(\Omega) \times \mathrm{K}(\Omega) .
$$

Remark 3.3. This result is the linear version of what is observed in the experiments: the lamellar phase is stable at high shear (see $[5,6])$. It is also what is observed numerically (see [3]).

As the most important point is the linear stability (Th. 3.2), we begin this section by proving this fact. We postone to the next paragraph the proof of Theorem 3.1.

\subsection{Linear stability}

In this section, we apply the stabilization process described in Section 2.3 in order to obtain bounded in time solutions, provided $\lambda$ is small enough.

This result need energy estimates on the following quantity: if $(v, h)$ is a solution of $(10-13)$ defined on $[0, T]$, sufficently smooth, let

$$
N(t)=|v|_{2}^{2}+E|h|_{2}^{2}+\left|\partial_{x}^{2} h\right|_{2}^{2}+\lambda\left|\partial_{z} h\right|_{2}^{2}+F\left(|V|_{2}^{2}+\left|\partial_{z} H\right|_{2}^{2}\right)
$$

and

$$
\tilde{N}(t)=N(t)-C \sqrt{\lambda} \int_{\Omega} \partial_{x} h \partial_{z} h
$$

where $C, E$ and $F$ are constants that will be precised later on, $V(t, z)=\frac{1}{2} \int_{-1}^{1} v(t, x, z) \mathrm{d} x, \partial_{z} H(t, z)=$ $\frac{1}{2} \int_{-1}^{1} \partial_{z} h(t, x, z) \mathrm{d} x$ are the mean values with respect to $x$ of $v$ and $\partial_{z} h$ respectively. 
Remark 3.4. We have to introduce in this Lyapounov function the quantities $V$ and $H$ (whereas it has not been the case in Sect. 2.3) because we can not prove a priori that the mean value of $v$ and $h$ are constant, contrary to the exemple in Section 2.3.

The proof of Theorem 3.2 will follow easily from this proposition:

Proposition 3.5. Let $E>4, F>\max \left(\frac{E}{\pi^{4}}, \frac{E}{\pi^{2}}\right)$ and $C<\min \left(\frac{1}{2}, \frac{2}{C_{p}}\right)$. Then, there exist $A>0$ and $\lambda_{0}>0$ such that, for all $\lambda<\lambda_{0}$,

$$
\tilde{N}^{\prime}(t) \leqslant-A N(t)
$$

The scheme of the proof will be:

- expansion in Fourier series;

- analogous result of (14) is found for large frequencies;

- then for small frequencies;

- finally for the mean value.

\subsubsection{Expansion in Fourier series}

As in Section 2.3, we expand the equations $(10,11)$ in Fourier series with respect to $x$, and we get

$$
\begin{aligned}
& \partial_{t} v+\frac{i k z}{\lambda} v=-k^{2} v+\partial_{z}^{2} v+\partial_{z}^{2} h-k^{2}\left(k^{2}-\delta_{1}\right) h \\
& \partial_{t} h+\frac{i k z}{\lambda} h=v+\partial_{z}^{2} h-k^{2}\left(k^{2}-\delta_{2}\right) h .
\end{aligned}
$$

We note $\Omega_{z}=(0,1)$. For sake of simplicity, $v$ still denotes $\hat{v}(k)=\frac{1}{2} \int_{-1}^{1} v(s) \exp (-i \pi k s) \mathrm{d} s$ and $h$ denotes $\hat{h}(k)$. Let denote, for $k \neq 0$,

and

$$
\begin{gathered}
N_{k}(t)=|v|_{2}^{2}+E|h|_{2}^{2}+k^{4}|h|_{2}^{2}+\lambda\left|\partial_{z} h\right|_{2}^{2}, \\
\tilde{N}_{k}(t)=N_{k}(t)-C k \sqrt{\lambda} \operatorname{Im} \int_{\Omega_{z}} h \partial_{z} \bar{h}
\end{gathered}
$$

$$
\tilde{N}_{0}(t)=N_{0}(t)=|v|_{2}^{2}+E|h|_{2}^{2}+\lambda\left|\partial_{z} h\right|_{2}^{2}+F\left(|v|_{2}^{2}+\left|\partial_{z} h\right|_{2}^{2}\right)
$$

We compute $\tilde{N}_{k}^{\prime}(t)$ and $\tilde{N}_{0}^{\prime}(t)$ :

$$
\frac{1}{2} \partial_{t}|v|_{2}^{2}=-k^{2}|v|_{2}^{2}-\left|\partial_{z} v\right|_{2}^{2}-\operatorname{Re} \int_{\Omega_{z}} \partial_{z} h \partial_{z} \bar{v}-k^{2}\left(k^{2}-\delta_{1}\right) \operatorname{Re} \int_{\Omega_{z}} h \bar{v}
$$

as $\operatorname{Re} \int_{\Omega_{z}} i z v \bar{v}=\operatorname{Re} \int_{\Omega_{z}} i z|v|^{2}=0$. The same computation gives:

$$
\begin{aligned}
& \frac{1}{2} \partial_{t}|h|_{2}^{2}=\operatorname{Re} \int_{\Omega_{z}} h \bar{v}-\left|\partial_{z} h\right|_{2}^{2}-k^{2}\left(k^{2}-\delta_{2}\right)|h|_{2}^{2} . \\
\frac{1}{2} \partial_{t}\left|\partial_{z} h\right|_{2}^{2}= & -\operatorname{Re} \int_{\Omega_{z}} \frac{-i z k}{\lambda} h \partial_{z}^{2} \bar{h}-\operatorname{Re} \int_{\Omega_{z}} v \partial_{z}^{2} \bar{h}-\left|\partial_{z}^{2} h\right|_{2}^{2}-k^{2}\left(k^{2}-\delta_{2}\right)\left|\partial_{z} h\right|_{2}^{2} \\
= & \frac{k}{\lambda} \operatorname{Im} \int_{\Omega_{z}} h \partial_{z} \bar{h}+\operatorname{Re} \int_{\Omega_{z}} \partial_{z} v \partial_{z} \bar{h}-\left|\partial_{z}^{2} h\right|_{2}^{2}-k^{2}\left(k^{2}-\delta_{2}\right)\left|\partial_{z} h\right|_{2}^{2},
\end{aligned}
$$


by integrating by parts the imaginary term.

$$
\begin{aligned}
\frac{1}{2} \partial_{t} \operatorname{Im} \int_{\Omega_{z}} h \partial_{z} \bar{h}= & \operatorname{Im} \int_{\Omega_{z}} \frac{-i k z}{\lambda} h \partial_{z} \bar{h}+\operatorname{Im} \int_{\Omega_{z}} v \partial_{z} \bar{h}+\int_{\Omega_{z}} \partial_{z}^{2} h \partial_{z} \bar{h} \\
& -k^{2}\left(k^{2}-\delta_{2}\right) \operatorname{Im} \int_{\Omega_{z}} h \partial_{z} \bar{h}
\end{aligned}
$$

Integrating by parts the first term of the right member gives

$$
\frac{1}{2} \partial_{t} \operatorname{Im} \int_{\Omega_{z}} h \partial_{z} \bar{h}=\frac{k}{2 \lambda}|h|_{2}^{2}+\operatorname{Im} \int_{\Omega_{z}} v \partial_{z} \bar{h}+\operatorname{Im} \int_{\Omega_{z}} \partial_{z}^{2} h \partial_{z} \bar{h}-k^{2}\left(k^{2}-\delta_{2}\right) \operatorname{Im} \int_{\Omega_{z}} h \partial_{z} \bar{h} .
$$

As in Section 2.3, we study separately high frequencies and small ones. Due to its special behaviour, $\tilde{N}_{0}$ is treated separately. By using (17-20) for $k \neq 0$, we have

$$
\begin{aligned}
\frac{1}{2} \tilde{N}_{k}^{\prime}(t)= & -k^{2}|v|_{2}^{2}-\left|\partial_{z} v\right|_{2}^{2}+\delta_{1} k^{2} \operatorname{Re} \int_{\Omega_{z}} h \bar{v}+E \operatorname{Re} \int_{\Omega_{z}} h \bar{v}-E\left|\partial_{z} h\right|_{2}^{2} \\
& -E k^{2}\left(k^{2}-\delta_{2}\right)|h|_{2}^{2}-k^{4}\left|\partial_{z} h\right|_{2}^{2}-k^{6}\left(k^{2}-\delta_{2}\right)|h|_{2}^{2} \\
& +k \operatorname{Im} \int_{\Omega_{z}} h \overline{\partial_{z} h}+\lambda \operatorname{Re} \int_{\Omega_{z}} \partial_{z} v \overline{\partial_{z} h}-\lambda\left|\partial_{z}^{2} h\right|_{2}^{2} \\
& -\lambda k^{2}\left(k^{2}-\delta_{2}\right)\left|\partial_{z} h\right|_{2}^{2}-\frac{C k^{2}}{2 \sqrt{\lambda}}|h|_{2}^{2}-C k \sqrt{\lambda} \operatorname{Im} \int_{\Omega_{z}} v \overline{\partial_{z} h} \\
& -C k \sqrt{\lambda} \operatorname{Im} \int_{\Omega_{z}} \partial_{z}^{2} h \overline{\partial_{z} h}-C \sqrt{\lambda} k^{3}\left(k^{2}-\delta_{2}\right) \operatorname{Im} \int_{\Omega_{z}} h \overline{\partial_{z} h}
\end{aligned}
$$

and, using (17-19), for $k=0$,

$$
\begin{aligned}
\frac{1}{2} \tilde{N}_{0}^{\prime}(t)= & -\left|\partial_{z} v\right|_{2}^{2}+E \operatorname{Re} \int_{\Omega_{z}} h \bar{v}-E\left|\partial_{z} h\right|_{2}^{2}+\lambda \operatorname{Re} \int_{\Omega_{z}} \partial_{z} v \overline{\partial_{z} h} \\
& -\lambda\left|\partial_{z}^{2} h\right|_{2}^{2}-F\left|\partial_{z} v\right|_{2}^{2}-F\left|\partial_{z}^{2} h\right|_{2}^{2}
\end{aligned}
$$

Let note that for this last equation, $V$ is equal to $v$ (let recall that $v$ means $\hat{v}(0)$ ) and is a real function. The same remarks hold for $h$.

\subsubsection{High frequencies}

As in Section 2.3, the main idea if that, for $k$ large enough, the viscous terms are dominant. By using the fact that the sign of a quadratic form does not change if its discriminant is negative, we take each term of (21) which is not negative and find an associated quadratic form which controls them for large $k$. Hence, we get the following result for linear stability for large frequencies:

Proposition 3.6. Let $\Lambda>0$, then there exists $K$ in $\mathbb{N}$, depending only on $\Lambda, C$ and $E$, and $A_{1}>0$ such that, for all $\lambda$ in $(0, \Lambda)$, for all $k>K$ and for all $t$ in $[0, T), \tilde{N}_{k}^{\prime}(t) \leqslant-A_{1} N_{k}(t)$.

Proof. First, we choose $k$ sufficiently large so that $k^{2}-\delta_{2}$ is positive.

1. For $\operatorname{Re} \int_{\Omega_{z}} h \bar{v}$ :

$$
Q_{1}=-\frac{k^{2}}{4}|v|_{2}^{2}+\left(\delta_{1} k^{2}+E\right) \operatorname{Re} \int_{\Omega_{z}} h \bar{v}-\frac{k^{6}}{8}\left(k^{2}-\delta_{2}\right)|h|_{2}^{2}
$$


Using Cauchy-Schwarz's inequality leads to

$$
Q_{1} \leqslant-\frac{k^{2}}{4}|v|_{2}^{2}+\left(\delta_{1} k^{2}+E\right)|v|_{2}|h|_{2}-\frac{k^{6}}{8}\left(k^{2}-\delta_{2}\right)|h|_{2}^{2} .
$$

The second member is a quadratic form whose discriminant is $\Delta_{1}=\left(\delta_{1} k^{2}+E\right)^{2}-\frac{k^{8}}{8}\left(k^{2}-\delta_{2}\right)$. So for $k$ sufficiently large, $\Delta_{1}<0$ and $Q_{1} \leqslant 0$. The five following quantities will be treated in the same way.

2. For $\operatorname{Re} \int_{\Omega_{z}} \partial_{z} h \partial_{z} \bar{v}$ :

$$
\begin{aligned}
Q_{2} & =-\frac{1}{2}\left|\partial_{z} v\right|_{2}^{2}+(\lambda-1) \operatorname{Re} \int_{\Omega_{z}} \partial_{z} h \partial_{z} \bar{v}-\frac{1}{8} k^{4}\left|\partial_{z} h\right|_{2}^{2}, \\
& \leqslant-\frac{1}{2}\left|\partial_{z} v\right|_{2}^{2}+(\lambda-1)\left|\partial_{z} h\right|_{2}\left|\partial_{z} v\right|_{2}-\frac{1}{8} k^{4}\left|\partial_{z} h\right|_{2}^{2} .
\end{aligned}
$$

The discriminant of the second member is $\Delta_{2}=(\lambda-1)^{2}-\frac{1}{4} k^{4}$. Let $\Lambda>0$. Then, for $k$ sufficiently large and for all $\lambda<\Lambda, \Delta_{2}<0$ and $Q_{2} \leqslant 0$.

3. For $\operatorname{Im} \int_{\Omega_{z}} h \partial_{z} \bar{h}:$

$$
\begin{aligned}
Q_{3} & =-\frac{k^{6}}{8}\left(k^{2}-\delta 2\right)|h|_{2}^{2}+k \operatorname{Im} \int_{\Omega_{z}} h \partial_{z} \bar{h}-\frac{k^{4}}{8}\left|\partial_{z} h\right|_{2}^{2} \\
& \leqslant-\frac{k^{6}}{8}\left(k^{2}-\delta 2\right)|h|_{2}^{2}+k|h|_{2}^{2}\left|\partial_{z} h\right|_{2}^{2}-\frac{k^{4}}{8}\left|\partial_{z} h\right|_{2}^{2} .
\end{aligned}
$$

The discriminant of the second member is $\Delta_{3}=k^{2}-\frac{1}{16} k^{10}\left(k^{2}-\delta 2\right)$, so that for $k$ large enough, $\Delta_{3}<0$ and $Q_{3} \leqslant 0$.

4. For $k \sqrt{\lambda} \operatorname{Im} \int_{\Omega_{z}} v \partial_{z} \bar{h}:$

$$
\begin{aligned}
Q_{4} & =-\frac{1}{4} k^{2}|v|_{2}^{2}-C k \sqrt{\lambda} \operatorname{Im} \int_{\Omega_{z}} v \partial_{z} \bar{h}-\frac{\lambda}{4} k^{2}\left(k^{2}-\delta_{2}\right)\left|\partial_{z} h\right|_{2}^{2} \\
& \leqslant-\frac{1}{4} k^{2}|v|_{2}^{2}+C k \sqrt{\lambda}|v|_{2}^{2}\left|\partial_{z} h\right|_{2}^{2}-\frac{\lambda}{4} k^{2}\left(k^{2}-\delta_{2}\right)\left|\partial_{z} h\right|_{2}^{2} .
\end{aligned}
$$

The discriminant of the right member is $\Delta_{4}=\lambda k^{2}\left(C^{2}-\frac{1}{4} k^{2}\left(k^{2}-\delta_{2}\right)\right)$. So for $k$ sufficiently large, $\Delta_{4}<0$ and $Q_{4} \leqslant 0$.

5. For $k \sqrt{\lambda} \operatorname{Im} \int_{\Omega_{z}} \partial_{z}^{2} h \partial_{z} \bar{h}:$

$$
\begin{aligned}
Q_{5} & =-\frac{\lambda}{2}\left|\partial_{z}^{2} h\right|_{2}^{2}-C k \sqrt{\lambda} \operatorname{Im} \int_{\Omega_{z}} \partial_{z}^{2} h \partial_{z} \bar{h}-\frac{1}{4} k^{4}\left|\partial_{z} h\right|_{2}^{2} \\
& \leqslant-\frac{\lambda}{2}\left|\partial_{z}^{2} h\right|_{2}^{2}+C k \sqrt{\lambda}\left|\partial_{z} h\right|_{2}^{2}\left|\partial_{z}^{2} h\right|_{2}^{2}-\frac{1}{4} k^{4}\left|\partial_{z} h\right|_{2}^{2}
\end{aligned}
$$

The discriminant of the second member is $\Delta_{5}=\lambda k^{2}\left(C^{2}-\frac{1}{2} k^{2}\right)$. So for $k$ large enough, $\Delta_{5}<0$ and $Q_{5} \leqslant 0$.

6. For $k^{3} \sqrt{\lambda}\left(k^{2}-\delta_{2}\right) \operatorname{Im} \int_{\Omega_{z}} h \partial_{z} \bar{h}$ :

$$
\begin{aligned}
Q_{6} & =\left(k^{2}-\delta_{2}\right)\left(-\frac{1}{4} k^{6}|h|_{2}^{2}+C k^{3} \sqrt{\lambda} \operatorname{Im} \int_{\Omega_{z}} h \partial_{z} \bar{h}-\frac{\lambda}{4} k^{2}\left|\partial_{z} h\right|_{2}^{2}\right) \\
& \leqslant k^{2}\left(k^{2}-\delta_{2}\right)\left(-\frac{1}{4} k^{4}|h|_{2}^{2}+C k \sqrt{\lambda}|h|_{2}^{2}\left|\partial_{z} h\right|_{2}^{2}-\frac{\lambda}{4}\left|\partial_{z} h\right|_{2}^{2}\right)
\end{aligned}
$$


The discriminant of the second member is $\Delta_{6}=\lambda k^{2}\left(C^{2}-\frac{1}{4} k^{2}\right)$. So for $k$ sufficiently large, $\Delta_{6}<0$ and $Q_{6} \leqslant 0$.

Then, using the fact that for $k$ large enough, $Q_{1}, Q_{2}, Q_{3}, Q_{4}, Q_{5}$ and $Q_{6}$ are non positive, we have

$$
\begin{aligned}
\tilde{N}^{\prime}(t)+k^{2}|v|_{2}^{2}+\left|\partial_{z} v\right|_{2}^{2}+\left(2 E k^{2}+k^{6}\right)\left(k^{2}-\delta_{2}\right)|h|_{2}^{2}+\frac{C k^{2}}{\sqrt{\lambda}}|h|_{2}^{2} & \\
& +\left(2 E+k^{4}+\lambda k^{2}\left(k^{2}-\delta_{2}\right)\right)\left|\partial_{z} h\right|_{2}^{2}+\lambda\left|\partial_{z}^{2} h\right|_{2}^{2} \leqslant 0 .
\end{aligned}
$$

As $\frac{1}{2} k^{2}-\delta_{2}>0$, for $k$ large enough,

$$
\begin{aligned}
\tilde{N}^{\prime}(t) \leqslant & -\frac{k^{2}}{2}\left(|v|_{2}^{2}+\left(E k^{2}+\frac{1}{2} k^{6}\right)|h|_{2}^{2}+\lambda k^{2}\left|\partial_{z} h\right|_{2}^{2}\right) \\
& -\frac{1}{2} k^{2}|v|_{2}^{2}-\left|\partial_{z} v\right|_{2}^{2}-\frac{1}{4} k^{4}\left(2 E-k^{4}\right)|h|_{2}^{2} \\
& -\frac{C k^{2}}{\sqrt{\lambda}}|h|_{2}^{2}-\left(2 E-k^{4}\right)\left|\partial_{z} h\right|_{2}^{2}-\lambda\left|\partial_{z}^{2} h\right|_{2}^{2} .
\end{aligned}
$$

Let $k$ large enough such that $k^{2}>1$ and $k^{2}\left(2 E+k^{4}\right)>2\left(E+k^{4}\right)$. Then there exists $K>0$ and $\mu$ (depending on $E$ and $C$ ) such that, for all $\lambda<\Lambda$ and for all $k>K$ we have

$$
\tilde{N}_{k}^{\prime}(t)+\mu\left(k^{2}|v|_{2}^{2}+\left|\partial_{z} v\right|_{2}^{2}+\left(k^{4}+k^{8}\right)|h|_{2}^{2}+\left|\partial_{z} h\right|_{2}^{2}+k^{4}\left|\partial_{z} h\right|_{2}^{2}\right)+\frac{C k^{2}}{\sqrt{\lambda}}|h|_{2}^{2}+\lambda\left|\partial_{z}^{2} h\right|_{2}^{2} \leqslant-\frac{K}{2} N_{k}(t)
$$

Hence the proof is finished by choosing $A_{1}=\frac{K}{2}$.

Remark 3.7. Let note that this result has a physical interpretation: high frequencies are always stable, so that the structures that are created by the instability and that are observed in the experiments have a size that corresponds to the small waves numbers.

\subsubsection{Low frequencies}

In this section, we study the frequencies $k$ between 1 and $K$, the case $k=0$ being studied in the next paragraph. Here, the viscous terms can not control the non negative terms of (21). We however have an $a$ priori estimates thanks to the term of order $\lambda^{-\frac{1}{2}}$. The method is strictly the same: we use the same property of quadratic forms as in the previous lemma.

Lemma 3.8. There exists $\lambda_{0}>0$ and $A_{2}>0$ depending on $E$ and $C$ such that, for all $\lambda$ in $\left(0, \lambda_{0}\right)$, for all $k$ between 1 and $K, \tilde{N}_{k}^{\prime}(t) \leqslant-A_{2} N_{k}(t)$.

Proof. As in the previous lemma, we control each non negative term by negative terms:

1. For $\operatorname{Re} \int_{\Omega_{z}} h \bar{v}$ :

$$
\begin{aligned}
Q_{1} & =-\frac{k^{2}}{4}|v|_{2}^{2}+\left(\delta_{1} k^{2}+E\right) \operatorname{Re} \int_{\Omega_{z}} h \bar{v}-\frac{C k^{2}}{16 \sqrt{\lambda}}|h|_{2}^{2} \\
& \leqslant-\frac{k^{2}}{4}|v|_{2}^{2}+\left(\delta_{1} k^{2}+E\right)|v|_{2}|h|_{2}-\frac{C k^{2}}{16 \sqrt{\lambda}}|h|_{2}^{2} .
\end{aligned}
$$

The second member is a quadratic form and its discriminant is $\Delta_{1}=\left(\delta_{1} k^{2}+E\right)^{2}-\frac{C k^{4}}{16 \sqrt{\lambda}}$. So for $\lambda$ small enough $\left(\lambda<\Lambda, \Lambda\right.$ fixed in Prop. 3.6) and for all $k \leqslant K, \Delta_{1}<0$ and $Q_{1} \leqslant 0$. 
2. For $\operatorname{Re} \int_{\Omega_{z}} \partial_{z} h \partial_{z} \bar{v}$ :

$$
\begin{aligned}
Q_{2} & =-\frac{1}{2}\left|\partial_{z} v\right|_{2}^{2}+(\lambda-1) \operatorname{Re} \int_{\Omega_{z}} \partial_{z} h \partial_{z} \bar{v}-\frac{E}{8}\left|\partial_{z} h\right|_{2}^{2} \\
& \leqslant-\frac{1}{2}\left|\partial_{z} v\right|_{2}^{2}+(\lambda-1)\left|\partial_{z} h\right|_{2}\left|\partial_{z} v\right|_{2}-\frac{E}{8}\left|\partial_{z} h\right|_{2}^{2}
\end{aligned}
$$

The second member is a quadratic form and its discriminant is $\Delta_{2}=(\lambda-1)^{2}-\frac{E}{4} \underset{\lambda \rightarrow 0}{\longrightarrow} 1-\frac{E}{4}<0$, by choice of $E$. So for $\lambda$ small enough and for all $k \leqslant K, \Delta_{2}<0$ and $Q_{2} \leqslant 0$.

3. For $\operatorname{Im} \int_{\Omega_{z}} h \partial_{z} \bar{h}$ :

$$
\begin{aligned}
Q_{3} & =-\frac{C k^{2}}{16 \sqrt{\lambda}}|h|_{2}^{2}+k \operatorname{Im} \int_{\Omega_{z}} h \partial_{z} \bar{h}-\frac{E}{8}\left|\partial_{z} h\right|_{2}^{2} \\
& \leqslant-\frac{C k^{2}}{16 \sqrt{\lambda}}|h|_{2}^{2}+k|h|_{2}^{2}\left|\partial_{z} h\right|_{2}^{2}-\frac{E}{8}\left|\partial_{z} h\right|_{2}^{2} .
\end{aligned}
$$

The second member is a quadratic form and its discriminant is $\Delta_{3}=k^{2}\left(1-\frac{C E}{32 \sqrt{\lambda}}\right)$, so that for all $k \leqslant K$ and for $\lambda$ small enough, $\Delta_{3}<0$ and $Q_{3} \leqslant 0$.

4. For $k \sqrt{\lambda} \operatorname{Im} \int_{\Omega_{z}} v \partial_{z} \bar{h}:$

$$
\begin{aligned}
Q_{4} & =-\frac{1}{4} k^{2}|v|_{2}^{2}-C k \sqrt{\lambda} \operatorname{Im} \int_{\Omega_{z}} v \partial_{z} \bar{h}-\frac{E}{4}\left|\partial_{z} h\right|_{2}^{2} \\
& \leqslant-\frac{1}{4} k^{2}|v|_{2}^{2}+C k \sqrt{\lambda}|v|_{2}^{2}\left|\partial_{z} h\right|_{2}^{2}-\frac{E}{4}\left|\partial_{z} h\right|_{2}^{2}
\end{aligned}
$$

The second member is a quadratic form and its discriminant is $\Delta_{4}=k^{2}\left(C^{2} \lambda-\frac{E}{4}\right)$, so that for all $k \leqslant K$ and for $\lambda$ small enough, $\Delta_{4}<0$ and $Q_{4} \leqslant 0$.

5. For $k \sqrt{\lambda} \operatorname{Im} \int_{\Omega_{z}} \partial_{z}^{2} h \partial_{z} \bar{h}$ :

$$
\begin{aligned}
Q_{5} & =-\frac{\lambda}{2}\left|\partial_{z}^{2} h\right|_{2}^{2}-C k \sqrt{\lambda} \operatorname{Im} \int_{\Omega_{z}} \partial_{z}^{2} h \partial_{z} \bar{h}-\frac{1}{8} k^{4}\left|\partial_{z} h\right|_{2}^{2} \\
& \leqslant-\frac{\lambda}{2}\left|\partial_{z}^{2} h\right|_{2}^{2}+C k \sqrt{\lambda}\left|\partial_{z} h\right|_{2}^{2}\left|\partial_{z}^{2} h\right|_{2}^{2}-\frac{1}{8} k^{4}\left|\partial_{z} h\right|_{2}^{2}
\end{aligned}
$$

The second member is a quadratic form and its discriminant is $\Delta_{5}=\lambda k^{2}\left(C^{2}-\frac{1}{4} k^{2}\right.$ ) (as we choose $C<\frac{1}{2}$ ), so that for all $k \leqslant K$ and for $\lambda$ small enough, $\Delta_{5}<0$ and $Q_{5} \leqslant 0$.

6. For $k^{3} \sqrt{\lambda}\left(k^{2}-\delta_{2}\right) \operatorname{Im} \int_{\Omega_{z}} h \partial_{z} \bar{h}$ :

$$
\begin{aligned}
Q_{6} & =-\frac{1}{8} k^{4}\left|\partial_{z} h\right|_{2}^{2}+C k^{3} \sqrt{\lambda}\left(k^{2}-\delta_{2}\right) \operatorname{Im} \int_{\Omega_{z}} h \partial_{z} \bar{h}-\frac{C k^{2}}{16 \sqrt{\lambda}}|h|_{2}^{2} \\
& \leqslant-\frac{1}{8} k^{4}\left|\partial_{z} h\right|_{2}^{2}+C k^{3} \sqrt{\lambda}\left(k^{2}-\delta_{2}\right)|h|_{2}^{2}\left|\partial_{z} h\right|_{2}^{2}-\frac{C k^{2}}{16 \sqrt{\lambda}}|h|_{2}^{2}
\end{aligned}
$$


The second member is a quadratic form and its discriminant is

$$
\Delta_{6}=C^{2} \lambda k^{6}\left(k^{2}+\delta_{2}\right)^{2}-\frac{C k^{6}}{32 \sqrt{\lambda}} \leqslant C^{2} \lambda K^{6}\left(K^{2}+\delta_{2}\right)^{2}-\frac{C}{32 \sqrt{\lambda}},
$$

so that for all $k \leqslant K$ and for $\lambda$ small enough, $\Delta_{6}<0$ and $Q_{6} \leqslant 0$.

By using the fact that, for $\lambda$ small enough and for all $k \leqslant K, Q_{1}, \ldots, Q_{6}$ are non positive, we have

$$
\begin{aligned}
\tilde{N}_{k}^{\prime}(t) \leqslant-k^{2}|v|_{2}^{2}-\left|\partial_{z} v\right|_{2}^{2}-2 k^{2}\left(k^{2}-\delta_{2}\right)\left(E+k^{4}\right)|h|_{2}^{2} & -\frac{3 C k^{2}}{4 \sqrt{\lambda}}|h|_{2}^{2} \\
& -\left(E+\frac{3}{2} k^{4}\right)\left|\partial_{z} h\right|_{2}^{2}-2 \lambda k^{2}\left(k^{2}-\delta_{2}\right)\left|\partial_{z} h\right|_{2}^{2}-\lambda\left|\partial_{z}^{2} h\right|_{2}^{2} .
\end{aligned}
$$

It remains some terms (in $|h|_{2}^{2}$ and in $\left|\partial_{z} h\right|_{2}^{2}$ ) which are not necessarily negative. As usual, we control them by using the term of order $\lambda^{-\frac{1}{2}}$ :

For $\lambda$ small enough, we have $\frac{C}{8 \sqrt{\lambda}}>2 E \delta_{2}+2 \delta_{2} K^{4}$, then, for all $k \leqslant K$,

$$
2 E \delta_{2} k^{2}+2 \delta_{2} k^{6}-\frac{C k^{2}}{8 \sqrt{\lambda}} \leqslant 0
$$

For $\lambda$ small enough, we have $2 \lambda \delta_{2}<\frac{1}{2}$, then, for all $k \leqslant K, 2 \lambda \delta_{2} k^{2}-\frac{1}{2} \gamma_{2} k^{4}<0$.

Hence, there exists $\mu>0$ such that for $\lambda$ small enough, for all $k \leqslant K$,

$$
\begin{aligned}
\tilde{N}_{k}^{\prime}(t)+\mu\left(k^{2}|v|_{2}^{2}+\left|\partial_{z} v\right|_{2}^{2}+\left(k^{4}+k^{8}+\frac{C k^{2}}{2 \sqrt{\lambda}}\right)|h|_{2}^{2}\right. & \left.+\left(1+k^{4}\right)\left|\partial_{z} h\right|_{2}^{2}\right)+\lambda\left|\partial_{z}^{2} h\right|_{2}^{2} \leqslant \\
& -\frac{1}{2} k^{2}|v|_{2}^{2}-E k^{4}|h|_{2}^{2}-k^{8}|h|_{2}^{2}-\frac{C k^{2}}{8 \sqrt{\lambda}}|h|_{2}^{2}-2 \lambda k^{4}\left|\partial_{z} h\right|_{2}^{2} .
\end{aligned}
$$

It is easy to show that, for $\lambda$ small enough and for all $k$ in $\{1, \ldots, K\}$, the right member of this inequality is less or equal than $-A_{2} N_{k}(t)$, for some $A_{2}>0$ depending on $E, C$ and $\lambda_{0}$.

Then, we have the following result:

$$
\tilde{N}_{k}^{\prime}(t)+\mu\left(k^{2}|v|_{2}^{2}+\left|\partial_{z} v\right|_{2}^{2}+\left(k^{4}+k^{8}+\frac{C k^{2}}{2 \sqrt{\lambda}}\right)|h|_{2}^{2}+\left(1+k^{4}\right)\left|\partial_{z} h\right|_{2}^{2}\right)+\lambda\left|\partial_{z}^{2} h\right|_{2}^{2} \leqslant-A_{2} N_{k}(t)
$$

which obviously concludes the proof.

\subsubsection{The mean value}

As $N_{0}$ (and of course $\tilde{N}_{0}$ ) has a different form than $N_{k}$, for $k \neq 0$, we study this case here and we have the following lemma:

Lemma 3.9. There exists $\lambda_{0}>0$ and $A_{3}>0$ such that, for all $\lambda$ in $\left(0, \lambda_{0}\right), \tilde{N}_{0}^{\prime}(t) \leqslant-A_{3} N_{0}(t)$.

Proof. This result is based on the symmetry of the system. Indeed, the evolution of $|v|_{2}$ and $\left|\partial_{z} h\right|_{2}$ is given by

$$
\frac{1}{2} \partial_{t}|v|_{2}^{2}=-\left|\partial_{z} v\right|_{2}^{2}-\operatorname{Re} \int_{\Omega_{z}} \partial_{z} h \partial_{z} \bar{v}
$$


and

$$
\frac{1}{2} \partial_{t}\left|\partial_{z} h\right|_{2}^{2}=\operatorname{Re} \int_{\Omega_{z}} \partial_{z} h \partial_{z} \bar{v}-\left|\partial_{z}^{2} h\right|_{2}^{2} .
$$

It is obvious that by taking a linear combination, we eliminate the $\operatorname{Re} \int_{\Omega_{z}} \partial_{z} h \partial_{z} \bar{v}$ term, allowing us to control $|v|_{2}^{2}$ and $\left|\partial_{z} h\right|_{2}^{2}$ and the other terms of $N_{0}$. As $v$ and $h$ verify $|v|_{2} \leqslant C_{p}\left|\partial_{z} v\right|_{2},|h|_{2} \leqslant C_{p}^{2}\left|\partial_{z}^{2} h\right|_{2}$ and $\left|\partial_{z} h\right|_{2} \leqslant C_{p}\left|\partial_{z}^{2} h\right|_{2}$, the choice of $F$ and the fact that $\lambda_{0}$ can be as small as we want (here $\left.\lambda_{0}<1\right)$ imply that (22) becomes:

$$
\tilde{N}_{0}^{\prime}(t)+\frac{1}{2}\left|\partial_{z} v\right|_{2}^{2}+E\left|\partial_{z} h\right|_{2}^{2}+(\lambda+1)\left|\partial_{z}^{2} h\right|_{2}^{2} \leqslant-A_{3} N_{0}(t)
$$

where $A_{3}$ is a positif real. The proof is finished.

\subsubsection{Conclusion}

To establish the stability result, we need the following embedding lemma:

Lemma 3.10. Let $\mathrm{H}_{1} \hookrightarrow \mathrm{H} \hookrightarrow \mathrm{H}_{2}$ three hilbert spaces so that the first embedding is compact. Then for any $p>1$, the embedding

is compact.

$$
\left\{f \in \mathrm{L}^{\infty}\left(0, T ; \mathrm{H}_{1}\right), \frac{\mathrm{d} f}{\mathrm{~d} t} \in \mathrm{L}^{p}\left(0, T ; \mathrm{H}_{2}\right)\right\} \hookrightarrow \mathrm{C}^{0}([0, T] ; H)
$$

(For a proof, see [12], p. 85.) This lemma will also be used in the proof of Theorems 2.2 and 2.4.

Proof of Theorem 3.2. By Proposition 3.6, Lemmas 3.8 and 3.9, choosing $A=\min \left(A_{1}, A_{2}, A_{3}\right)$ implies that for all $\lambda$ in $\left(0, \lambda_{0}\right)$ and for all $k \geqslant 0$, then $\tilde{N}_{k}^{\prime}(t) \leqslant-A N_{k}(t)$. By taking the conjugate expression of (15) and (16), we have the same result for $k<0$ (the proof is exactly the same). The choice of $C$ implies that $\tilde{N}_{k}(t)$ and $N_{k}(t)$ are equivalent. Then, by adding the last inequality for $k$ in $\mathbb{Z}$ and by using the Parseval-Bessel's identity, one get $\tilde{N}^{\prime}(t) \leqslant-A \tilde{N}(t)$.

More precisely, using inequalities (23-25), for some $B>0$, we have

$$
\tilde{N}^{\prime}(t)+B\left(\left|\partial_{x} v\right|_{2}^{2}+\left|\partial_{z} v\right|_{2}^{2}+\left|\partial_{x}^{2} h\right|_{2}^{2}+\left|\partial_{x}^{4} h\right|_{2}^{2}+\frac{1}{\sqrt{\lambda}}\left|\partial_{x} h\right|_{2}^{2}+\left|\partial_{z} h\right|_{2}^{2}+\left|\partial_{x}^{2} \partial_{z} h\right|_{2}^{2}+\lambda\left|\partial_{z}^{2} h\right|_{2}^{2}\right) \leqslant-A \tilde{N}(t)
$$

This inequality will be also used to perform the limit in the Galerkin method for the global in time existence theorem.

As the system is linear, the Galerkin method gives a sequence of approximated solution of (10-13), denoted $\left(v_{n}, h_{n}\right)$, which verify

$$
\begin{aligned}
& v_{n} \text { is in a bounded set of } \mathrm{L}^{\infty}\left(\mathbb{R}_{+} ; \mathrm{L}(\Omega)\right) \cap \mathrm{L}^{2}\left(\mathbb{R}_{+} ; \mathrm{H}(\Omega)\right), \\
& h_{n} \text { is in a bounded set of } \mathrm{L}^{\infty}\left(\mathbb{R}_{+} ; \mathrm{K}(\Omega)\right) \cap \mathrm{L}^{2}\left(\mathbb{R}_{+} ; \mathrm{H}^{2}(\Omega) \cap \mathrm{H}(\Omega)\right) .
\end{aligned}
$$

Then there exists

and

$$
v \in \mathrm{L}^{\infty}\left(\mathbb{R}_{+} ; \mathrm{L}(\Omega)\right) \cap \mathrm{L}^{2}\left(\mathbb{R}_{+} ; \mathrm{H}(\Omega)\right)
$$

such that

$$
h \in \mathrm{L}^{\infty}\left(\mathbb{R}_{+} ; \mathrm{K}(\Omega)\right) \cap \mathrm{L}^{2}\left(\mathbb{R}_{+} ; \mathrm{H}^{2}(\Omega) \cap \mathrm{H}(\Omega)\right)
$$

$$
\begin{aligned}
v_{n} \rightarrow & v \text { weak } * \text { in } \mathrm{L}^{\infty}\left(\mathbb{R}_{+} ; \mathrm{L}(\Omega)\right) \text { and weakly in } \mathrm{L}^{2}\left(\mathbb{R}_{+} ; \mathrm{H}(\Omega)\right), \\
h_{n} \rightarrow & h \text { weak } * \text { in } \mathrm{L}^{\infty}\left(\mathbb{R}_{+} ; \mathrm{K}(\Omega)\right) \text { and } \\
& \text { weakly in } \mathrm{L}^{2}\left(\mathbb{R}_{+} ; \mathrm{H}^{2}(\Omega) \cap \mathrm{H}(\Omega)\right) .
\end{aligned}
$$


The system being linear, it is straightforward to conclude that $(v, h)$ is a solution. We now deal with the fact that $(v, h)(t)$ tends to 0 . The proof is the same for $v$ and $h$, so we only show the result for $v$. It is easy to see that $\partial_{t} v_{n}$ and $\partial_{t} h_{n}$ are bounded in $\mathrm{L}^{2}\left(0, T ; \mathrm{H}^{-1}\right)$. So Lemma 3.10 implies that

$$
\left(v_{n}, h_{n}\right) \rightarrow(v, h) \text { in } \mathrm{C}^{0}\left([0, T] ; \mathrm{L}^{2}(\Omega) \times \mathrm{L}^{2}(\Omega)\right)
$$

Let $\epsilon>0$. There exists $T>0$ such that, for all $t>T$,

$$
\left\|v_{n}(t)\right\|_{\mathrm{L}(\Omega)} \leqslant N(0) \exp (-A t)<\frac{\epsilon}{2}
$$

The fact that this a priori estimate is independant of $n$ is the most important argument. Let $t>T$. Then (27) implies that

$$
\sup _{[0, t+1]}\left\|v_{n}-v\right\|_{\mathrm{L}(\Omega)} \underset{n \rightarrow+\infty}{\longrightarrow} 0
$$

i.e. there exists $n$ such that $\left\|v_{n}-v\right\|_{\mathrm{L}(\Omega)}<\frac{\epsilon}{2}$. So

$$
\|v(t)\|_{\mathrm{L}(\Omega)} \leqslant\left\|v_{n}-v\right\|_{\mathrm{L}(\Omega)}+\left\|v_{n}(t)\right\|_{\mathrm{L}(\Omega)} \leqslant \frac{\epsilon}{2}+N(0) \exp (-A t) \leqslant \epsilon .
$$

Remark 3.11. This result can be obtained without expandind $v$ and $h$ in Fourier series, but this method gives us a better understanding of the physical processes that are involved in the experiment:

- The symmetry of the system controls the mean value:

$$
\frac{1}{2} \partial_{t}\left(|V|_{2}^{2}+\left|\partial_{z} H\right|_{2}^{2}\right) \leqslant-\left|\partial_{z} V\right|_{2}^{2}-\left|\partial_{z}^{2} H\right|_{2}^{2}
$$

(cf. Lem. 3.9).

- The instabilities can not have a size corresponding to large waves numbers ( $c f$. Prop. 3.6).

- Small frequencies are controlled by the skew-symmetric operator $\frac{z}{\lambda} \partial_{x}$ when $\lambda$ is small (cf. Lem. 3.8), that is to say by the shear.

\subsection{Construction of the semi-group}

The aim of this paragraph is to prove Theorem 3.1. At this stage, we are not concerned with bounded in time solutions, so we do not try to apply the method described in Section 2.3.

Proof of Theorem 3.1. As usual, we work on some energy estimates. By computing $N(t)=|v|_{2}^{2}+\epsilon_{1}\left|\partial_{x}^{2} h\right|_{2}^{2}+\left|\partial_{z} h\right|_{2}^{2}$ with (10) and (11) we have

$$
\begin{aligned}
\frac{1}{2} N^{\prime}(t)= & -\left|\partial_{x} v\right|_{2}^{2}-\left|\partial_{z} v\right|_{2}^{2}-\int_{\Omega} \partial_{z} h \partial_{z} v+\delta_{1} \int_{\Omega} \partial_{x} h \partial_{x} v \\
& -\int_{\Omega} v \partial_{x}^{4} h+\int_{\Omega} v \partial_{x}^{4} h-\left|\partial_{x}^{2} \partial_{z} h\right|_{2}^{2} \\
& -\delta_{2} \int_{\Omega} \partial_{x}^{2} h \partial_{x}^{4} h-\left|\partial_{x}^{4} h\right|_{2}^{2}+\frac{1}{\lambda} \int_{\Omega} \partial_{x} h \partial_{z} h \\
& -\int_{\Omega} v \partial_{z}^{2} h-\left|\partial_{z}^{2} h\right|_{2}^{2}+\delta_{2} \int_{\Omega} \partial_{x}^{2} h \partial_{z}^{2} h-\left|\partial_{x}^{2} \partial_{z} h\right|_{2}^{2}
\end{aligned}
$$


Here, $C_{2}$ will be a constant. Using Young's inequality, one obtains the following inequalities:

- $-\int_{\Omega} \partial_{z} h \partial_{z} v \leqslant \frac{1}{2}\left|\partial_{z} v\right|_{2}^{2}+C_{2}\left|\partial_{z} h\right|_{2}^{2}$

- $\delta_{1} \int_{\Omega} \partial_{x} h \partial_{x} v \leqslant \frac{1}{2}\left|\partial_{x} v\right|_{2}^{2}+C_{2}\left|\partial_{x} h\right|_{2}^{2}$;

- $-\delta_{2} \int_{\Omega} \partial_{x}^{2} h \partial_{x}^{4} h \leqslant \frac{1}{2}\left|\partial_{x}^{4} h\right|_{2}^{2}+C_{2}\left|\partial_{x}^{2} h\right|_{2}^{2}$;

- $\frac{1}{\lambda} \int_{\Omega} \partial_{x} h \partial_{z} h \leqslant C_{2}\left|\partial_{x} h\right|_{2}^{2}+C_{2}\left|\partial_{z} h\right|_{2}^{2} \leqslant C_{2}\left|\partial_{x}^{2} h\right|_{2}^{2}+C_{2}\left|\partial_{z} h\right|_{2}^{2}$, by Poincaré's inequality;

- $-\int_{\Omega} v \partial_{z}^{2} h \leqslant \frac{1}{4}\left|\partial_{z}^{2} h\right|_{2}^{2}+C_{2}|v|_{2}^{2}$;

- $\delta_{2} \int_{\Omega} \partial_{x}^{2} h \partial_{z}^{2} h \leqslant \frac{1}{4}\left|\partial_{z}^{2} h\right|_{2}^{2}+C_{2}\left|\partial_{x}^{2} h\right|_{2}^{2}$.

By taking and $A=\max \left(C_{2}, \frac{1}{2}\right)$, we get

$$
N^{\prime}(t)+\left|\partial_{x} v\right|_{2}^{2}+\left|\partial_{z} v\right|_{2}^{2}+\left|\partial_{z}^{2} h\right|_{2}^{2}+\left|\partial_{x}^{2} \partial_{z} h\right|_{2}^{2}+\left|\partial_{x}^{4} h\right|_{2}^{2} \leqslant A N(t)
$$

which finishes the proof.

\section{NONLINEAR RESUltS}

We deal now with a priori estimates of the nonlinear terms, for both local and global in time cases. This last one require bounds on

Here is the result:

$$
N(t)=|v|_{2}^{2}+E|h|_{2}^{2}+\left|\partial_{x}^{2} h\right|_{2}^{2}+\lambda\left|\partial_{z} h\right|_{2}^{2}+F\left(|V|_{2}^{2}+\left|\partial_{z} H\right|_{2}^{2}\right) .
$$

Theorem 4.1. Let $E>4, F>\max \left(\frac{E}{\pi^{4}}, \frac{E}{\pi^{2}}\right)$ and $C<\min \left(\frac{1}{2}, \frac{2}{C_{p}}\right)$. Then there exists $\lambda_{0}, A>0, B>0$ (all depending on $E, F, C$ and the parameters of the system) and $Q$ a 2-valuated polynomial with positive coefficients, so that for all $(v, h)$ being a solution of the problem $(3,4)$ defined on $[0, T)$, for all $t$ in $[0, T)$ and for all $\lambda$ in $\left(0, \lambda_{0}\right)$,

$$
\tilde{N}^{\prime}(t)+B\left(\left|\partial_{x} v\right|_{2}^{2}+\left|\partial_{z} v\right|_{2}^{2}+\left|\partial_{x}^{2} h\right|_{2}^{2}+\left|\partial_{x}^{4} h\right|_{2}^{2}+\left|\partial_{z} h\right|_{2}^{2}+\left|\partial_{x}^{2} \partial_{z} h\right|_{2}^{2}+\lambda\left|\partial_{z}^{2} h\right|_{2}^{2}\right) \leqslant-A \tilde{N}(t)+Q(\tilde{N}(t)) .
$$

The local in time case can be interpreted as a particular form of the global one, as $\lambda$ does not play an important role and can be considered just as a physical parameter. Let

$$
N(t)=|v|_{2}^{2}+\left|\partial_{x}^{2} h\right|_{2}^{2}+\left|\partial_{z} h\right|_{2}^{2} .
$$

Then we have the

Theorem 4.2. Let $T>0$. Suppose that $(v, h)$ is a solution of the problem $(3,4)$. Then for all $\lambda>0$, there exists $A>0$ and $Q$ a 1-valuated polynomial with positive coefficients, so that for all $t \in[0, T)$,

$$
N^{\prime}(t)+A\left(\left|\partial_{x} v\right|_{2}^{2}+\left|\partial_{z} v\right|_{2}^{2}+\left|\partial_{x}^{4} h\right|_{2}^{2}+\left|\partial_{x}^{2} \partial_{z} h\right|_{2}^{2}+\left|\partial_{z}^{2} h\right|_{2}^{2}\right) \leqslant Q(N(t)) .
$$

Hence, we devote this section mainly to the proof of Theorem 4.1 which needs some inequalities we give in the next paragraph.

\subsection{Some inequalities}

The inequalities used to prove those theorems are Propositions 4.5 and 4.6 and its Corollary 4.7. Their proof are based on the Young's inequality (if $p, q>1, p^{-1}+q^{-1}=1, a, b \geqslant 0$ and $\epsilon>0$ then $a b \leqslant \epsilon a^{p}+b^{q} /(4 \epsilon)$ ) and the two following lemmas. Proposition 4.9 will be used later, to give compactness to the Galerkin sequence. 
Lemma 4.3. For all $p>1$, there exists $C>0$ such that, for all $u \in \mathrm{C}^{\infty}(\bar{B})$, 2-periodic in $x$, vanishing at $z=0,1$,

$$
|u|_{2 p}^{2 p} \leqslant C|u|_{2 p-2}^{2 p-2}|\nabla u|_{2}^{2} .
$$

The proof is based on the one given by Ladyzhenskaya in [9], which can be easily adapted for periodic functions.

Due to the anisotropic nature of the energy involved in the studied system $(1,2)$, we need the following Sobolev-type inequality:

Lemma 4.4. There exists $C>0$ such that for all $u \in \mathrm{C}^{\infty}(\bar{B})$, 2-periodic in $x$, vanishing at $z=0,1$ and for all $p \in[2,6]$,

$$
\left|\partial_{x} u\right|_{p} \leqslant C\left(\left|\partial_{x} u\right|_{2}+\left|\partial_{z} u\right|_{2}+\left|\partial_{x}^{2} u\right|_{2}\right)
$$

For a proof, $c f$. [1] (p. 323). This kind of inequality is also used for the K.P. equation in [4].

Proposition 4.5. For all $C_{1}>0$, there exists $C_{2}>0$ such that for all $u \in \mathrm{C}^{\infty}(\bar{\Omega})$, 2-periodic in $x$ and vanishing at $z=0,1$,

$$
\left|\partial_{z} u\right|_{4}^{3} \leqslant C_{2}\left|\partial_{z} u\right|_{2}^{6}+C_{1}\left(\left|\partial_{x}^{2} u\right|_{2}^{2}+\left|\partial_{z}^{2} u\right|_{2}^{2}\right) .
$$

Proof. First, the result of the Lemma 4.3 remains for $\partial_{z} u$ as its mean value with respect to $z$ is equal to zero. Then it suffices to reuse the arguments used for the $x$ variable. The Lemma 4.3 and Young's inequality leads to

$$
\begin{aligned}
& \left|\partial_{z} u\right|_{4}^{3} \leqslant C_{2}\left|\partial_{z} u\right|_{2}^{6}+C_{1}\left(\left|\partial_{x} \partial_{z} u\right|_{2}^{2}+\left|\partial_{z}^{2} u\right|_{2}^{2}\right) . \\
& \left|\partial_{x} \partial_{z} u\right|_{2}^{2}=\int_{\Omega} \partial_{x}^{2} u \partial_{z}^{2} u \leqslant \frac{1}{2}\left(\left|\partial_{x}^{2} u\right|_{2}^{2}+\left|\partial_{z}^{2} u\right|_{2}^{2}\right)
\end{aligned}
$$

by Cauchy-Schwartz's inequality. This concludes the proof.

Proposition 4.6. There exists $C>0$ such that for all $u \in \mathrm{C}^{\infty}(\bar{\Omega})$, 2-periodic in $x$ and vanishing at $z=0,1$ and for all $p \in[2,6]$,

$$
\left|\partial_{x} u\right|_{p} \leqslant C\left(\left|\partial_{z} u\right|_{2}+\left|\partial_{x}^{2} u\right|_{2}\right)
$$

Proof. We use the Lemma 4.4 and the Poincaré's inequality.

Corollary 4.7. There exists $C>0$ such that for all $u \in \mathrm{C}^{\infty}(\bar{\Omega})$, 2-periodic in $x$ and vanishing at $z=0,1$ and for all $p \in[2,6]$ and $q \geqslant 0$,

$$
\left|\partial_{x} u\right|_{p}^{2 q} \leqslant C\left(\left|\partial_{z} u\right|_{2}^{2}+\left|\partial_{x}^{2} u\right|_{2}^{2}\right)^{q}
$$

Proof. We use the Proposition 4.6 and the equivalence of norms in finite dimension.

Remark 4.8. By changing the constant $C$ of this corollary, we can adjust the coefficients of $\left|\partial_{z} u\right|_{2}^{2}$ and $\left|\partial_{x}^{2} u\right|_{2}^{2}$.

Proposition 4.9. There exists $C>0$ such that for all $u \in \mathrm{C}^{\infty}(\bar{\Omega})$, 2-periodic in $x$ and vanishing at $z=0,1$,

$$
\left|\partial_{x}^{2} u\right|_{4} \leqslant C\left|\partial_{x} u\right|_{4}^{\frac{1}{2}}\left|\partial_{x}^{2} u\right|_{2}^{\frac{1}{16}}\left|\partial_{x}^{4} u\right|_{2}^{\frac{1}{16}}\left|\partial_{x}^{3} u\right|_{6}^{\frac{3}{8}}
$$

Proof. Integrating by parts and using Hölder's inequality give

$$
\left|\partial_{x}^{2} u\right|_{4}^{4}=-3 \int_{\Omega} \partial_{z} u \partial_{x}^{3} u\left|\partial_{x}^{2} u\right|^{2} \leqslant 3\left|\partial_{x} u\right|_{4}\left|\partial_{x}^{3} u\right|_{4}\left|\partial_{x}^{2} u\right|_{4}^{2} .
$$

Classical results on interpolation inequalities give $\left|\partial_{x}^{3} u\right|_{4} \leqslant\left|\partial_{x}^{3} u\right|_{2}^{\frac{1}{4}}\left|\partial_{x}^{3} u\right|_{6}^{\frac{3}{4}}$. By integration by parts and using Cauchy-Schwartz's inequality we have $\left|\partial_{x}^{3} u\right|_{2} \leqslant\left|\partial_{x}^{2} u\right|_{2}^{\frac{1}{2}}\left|\partial_{x}^{4} u\right|_{2}^{\frac{1}{2}}$. This concludes the proof, with $C=\sqrt{3}$. 


\subsection{Nonlinear a priori estimates for global existence}

We complete in this paragraph the linear a priori estimate found in Section 3 (cf. Th. 3.2), by adding the one of nonlinear terms.

Basically, we will bound all nonlinear terms by polynomial in $N$, which will lead to the estimate of Theorem 4.1:

$$
\tilde{N}^{\prime}(t)+B\left(\left|\partial_{x} v\right|_{2}^{2}+\left|\partial_{z} v\right|_{2}^{2}+\left|\partial_{x}^{2} h\right|_{2}^{2}+\left|\partial_{x}^{4} h\right|_{2}^{2}+\left|\partial_{z} h\right|_{2}^{2}+\left|\partial_{x}^{2} \partial_{z} h\right|_{2}^{2}+\lambda\left|\partial_{z}^{2} h\right|_{2}^{2}\right) \leqslant-A \tilde{N}(t)+Q(\tilde{N}(t))
$$

where $Q$ is the polynomial.

Remark 4.10. - It is equivalent to work on $N(t)$ or $\tilde{N}(t)$ as they are equivalent.

- The fact that $Q$ is a 2-valuated polynomial is quite important: the linear part of the inequality of Theorem 4.1 as a negative coefficient. This is this fact that will give existence of the solution on $\mathbb{R}_{+}$.

- The others terms on the left-hand-side of this inequality are necessary to prove the convergence of the Galerkin sequence (see 5.3).

Theorem 4.1 is easily proved if the three nonlinear terms of (3) are bounded in a suitable way. The following proposition gives these bounds.

Proposition 4.11. Let $C_{1}>0$. Then there exists $C_{2}>0$ such that, for all $(v, h)$ being a solution of the problem (3, 4) and for $\mu<\lambda^{2}$ and $\nu^{2}<\lambda^{\frac{5}{2}}$,

$$
\begin{aligned}
-\frac{\mu}{\lambda} \int_{\Omega} \partial_{x} v \partial_{x} h \partial_{z} h-\frac{\mu}{\lambda} \int_{\Omega} \partial_{z} v\left(\partial_{x} h\right)^{2}+\frac{\nu^{2}}{\lambda} \int_{\Omega} \partial_{x} v\left(\partial_{x} h\right)^{3} & \leqslant C_{1}\left(\left|\partial_{x} v\right|_{2}^{2}+\left|\partial_{z} v\right|_{2}^{2}+\left|\partial_{x}^{2} h\right|_{2}^{2}+\lambda\left|\partial_{z}^{2} h\right|_{2}^{2}\right) \\
& +C_{2}\left(\left(\left|\partial_{x}^{2} h\right|_{2}^{2}+\lambda\left|\partial_{z} h\right|_{2}^{2}\right)^{2}+\left(\left|\partial_{x}^{2} h\right|_{2}^{2}+\lambda\left|\partial_{z} h\right|_{2}^{2}\right)^{3}\right) .
\end{aligned}
$$

Proof. We study separately the three terms of the left-hand-side.

- The $\int_{\Omega} \partial_{x} v \partial_{x} h \partial_{z} h$ term:

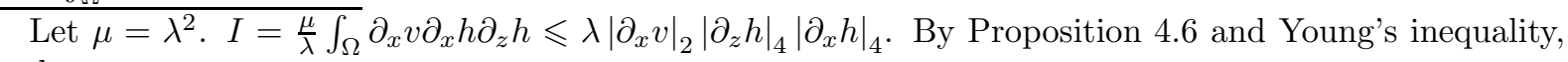
we have

With Proposition 4.5 and Corollary 4.7 we obtain:

$$
I \leqslant C_{1}\left|\partial_{x} v\right|_{2}^{2}+\lambda^{\frac{3}{2}}\left|\partial_{z} h\right|_{4}^{3}+C_{2} \lambda^{3}\left|\partial_{x} h\right|_{4}^{6} .
$$

$$
I \leqslant C_{1}\left(\left|\partial_{x} v\right|_{2}^{2}+\left|\partial_{x}^{2} h\right|_{2}^{2}+\lambda\left|\partial_{z}^{2} h\right|_{2}^{2}\right)+C_{2}\left(\lambda^{3}\left|\partial_{z} h\right|_{2}^{6}+\left(\left|\partial_{x}^{2} h\right|_{2}^{2}+\lambda\left|\partial_{z} h\right|_{2}^{2}\right)^{3}\right) .
$$

So, with the equivalence of norms in finite dimension:

$$
I \leqslant C_{1}\left(\left|\partial_{x} v\right|_{2}^{2}+\left|\partial_{x}^{2} h\right|_{2}^{2}+\lambda\left|\partial_{z}^{2} h\right|_{2}^{2}\right)+C_{2}\left(\left|\partial_{x}^{2} h\right|_{2}^{2}+\lambda\left|\partial_{z} h\right|_{2}^{2}\right)^{3} .
$$

- The $\int_{\Omega} \partial_{z} v\left(\partial_{x} h\right)^{2}$ term:

$$
I=\frac{\mu}{\lambda} \int_{\Omega} \partial_{z} v\left(\partial_{x} h\right)^{2} \leqslant \lambda\left|\partial_{z} v\right|_{2}\left|\partial_{x} h\right|_{4}^{2} \leqslant C_{1}\left|\partial_{z} v\right|_{2}^{2}+C_{2} \lambda^{2}\left|\partial_{x} h\right|_{4}^{4},
$$

by Young's inequality. By Corollary 4.7, we obtain:

$$
I \leqslant C_{1}\left|\partial_{z} v\right|_{2}^{2}+C_{2}\left(\lambda\left|\partial_{z} h\right|_{2}^{2}+\left|\partial_{x}^{2} h\right|_{2}^{2}\right)^{2} .
$$




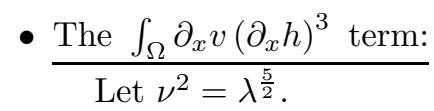

$$
I=\frac{\nu^{2}}{\lambda} \int_{\Omega} \partial_{x} v\left(\partial_{x} h\right)^{3} \leqslant \lambda^{\frac{3}{2}}\left|\partial_{x} v\right|_{2}\left|\partial_{x} h\right|_{6}^{3} \leqslant C_{1}\left|\partial_{x} v\right|_{2}^{2}+C_{2} \lambda^{3}\left|\partial_{x} h\right|_{6}^{6}
$$

by Young's inequality. With Corollary 4.7 we have:

$$
I \leqslant C_{1}\left|\partial_{x} v\right|_{2}^{2}+C_{2}\left(\left|\partial_{x}^{2} h\right|_{2}^{2}+\lambda\left|\partial_{z} h\right|_{2}^{2}\right)^{3}
$$

Proof of Theorem 4.1. Thanks to Theorem 3.2, we get (26) and $A_{1}, B$, and $\lambda_{0}$. Let $C_{1}<B$. Proposition 4.11 gives $C_{2}>0$ such that nonlinear terms are bounded by $C_{2}\left(N^{2}+N^{3}\right)$. So Theorem 4.1 is proved with $Q=C_{2}\left(X^{2}+X^{3}\right)$.

\subsection{Nonlinear a priori estimates for local existence}

The result for the local case needs few modifications. As it was said, $\lambda$ does not play any important role and is considered as a physical parameter. The proof is quite simple:

Proof of Theorem 4.2. The conclusion of Proposition 4.11 can be reused to obtain the result in combination to the inequality (28) of Theorem 3.1.

\section{Proof of the Global EXISTENCE}

In this section, we apply the Galerkin method (cf. [10]) to prove Theorem 2.2. This proof is completed in 4 points:

- we found a sequence of solutions of approximated PDE's of $(3,4)$. These solutions are denoted by $\left(v_{n}, h_{n}\right)$;

- we use a priori estimates found in Section 4 with $\left(v_{n}, h_{n}\right)$ to show that $v_{n}$ and $h_{n}$ are in a bounded set of some spaces;

- we show then the existence of a limit of a subsequence of $\left(v_{n}, h_{n}\right)$, denoted $(v, h)$, as well as their spatial derivatives. The difficult point is to show the strong convergence of nonlinecar terms;

- we conclude by the fact that $(v, h)$ is a solution of $(3,4)$.

\subsection{Approximation of the solution}

Let $e_{n, p}=\sin (n \pi z) \exp (i p \pi x)$ the usual complete orthonormal basis of $\mathrm{L}^{2}(\Omega)$, eigenvectors of $\Delta$ with boundary conditions used in this paper: periodic in $x$ and vanishing at $z=0,1$. For sake of simplicity, these eigenvectors will be indexed by $\mathbb{N}$. Let $n \in \mathbb{N}, E_{n}=\operatorname{Span}\left(e_{0}, \ldots, e_{n}\right)$ and $\Pi_{n}$ the orthogonal projector on $E_{n}$. Let $v_{n}=\sum_{i=0}^{n} a_{i}(t) e_{i}, h_{n}=\sum_{i=0}^{n} b_{i}(t) e_{i}$, where $a_{i}$ and $b_{i}$ are smooth real valued functions, $v_{0} \in \mathrm{L}(\Omega)$ and $h_{0} \in \mathrm{K}(\Omega)$.

So, we can note $\Pi_{n}\left(v_{0}\right)=\sum_{i=0}^{n} a_{i}^{0} e_{i}$ and $\Pi_{n}\left(h_{0}\right)=\sum_{i=0}^{n} b_{i}^{0} e_{i}$, where $a_{i}^{0}$ and $b_{i}^{0}$ are real constants. 
We consider the Galerkin approximation of (3) and (4):

$$
\begin{aligned}
& \partial_{t} v_{n}+\frac{1}{\lambda} \Pi_{n}\left(z \partial_{x} v_{n}\right)= \Delta v_{n}+\partial_{z}^{2} h_{n}-\delta_{1} \partial_{x}^{2} h_{n}-\partial_{x}^{4} h_{n} \\
&+\Pi_{n}\left(\frac{\mu}{\lambda} \partial_{x}\left(\partial_{x} h_{n} \partial_{z} h_{n}\right)+\frac{\mu}{\lambda} \partial_{z}\left(\partial_{x} h_{n}\right)^{2}+\frac{\nu^{2}}{\lambda} \partial_{x}\left(\partial_{x} h_{n}\right)^{3}\right) \\
& \partial_{t} h_{n}+\frac{1}{\lambda} \Pi_{n}\left(z \partial_{x} h_{n}\right)=v_{n}+\partial_{z}^{2} h_{n}-\delta_{2} \partial_{x}^{2} h_{n}+\partial_{x}^{4} h_{n},
\end{aligned}
$$

with the following initial conditions:

$$
v_{n}(0)=\Pi_{n}\left(v_{0}\right)
$$

and

$$
h_{n}(0)=\Pi_{n}\left(h_{0}\right) .
$$

Let $j \in\{0, \ldots, n\}$; we multiply (29) and (30) by $e_{j}$, then, since $\left(e_{n}\right)_{n \in \mathbb{N}}$ is an orthonormal system and $\Pi_{n}$ is self-adjoint,

$$
\begin{aligned}
\partial_{t} a_{j}+\frac{1}{\lambda} \sum_{k=0}^{n} a_{k} \int_{\Omega} z e_{j} \partial_{x} e_{k}= & \sum_{k=0}^{n} a_{k} \int_{\Omega} e_{j} \partial_{x}^{2} e_{k}+\sum_{k=0}^{n} a_{k} \int_{\Omega} e_{j} \partial_{z}^{2} e_{k} \\
& +\sum_{k=0}^{n} b_{k} \int_{\Omega} e_{j} \partial_{z}^{2} e_{k}-\delta_{1} \sum_{k=0}^{n} b_{k} \int_{\Omega} e_{j} \partial_{x}^{2} e_{k} \\
& +\sum_{k=0}^{n} b_{k} \int_{\Omega} e_{j} \partial_{x}^{4} e_{k}-F\left(\frac{\mu}{\lambda}, \frac{\mu}{\lambda}, \frac{\nu}{\lambda}\right)(X) \\
\partial_{t} b_{j}+\frac{1}{\lambda} \sum_{k=0}^{n} b_{k} \int_{\Omega} z e_{j} \partial_{x} e_{k}= & a_{j}+\sum_{k=0}^{n} b_{k} \int_{\Omega} e_{j} \partial_{z}^{2} e_{k}-\delta_{2} \sum_{k=0}^{n} b_{k} \int_{\Omega} e_{j} \partial_{x}^{2} e_{k} \\
& +\sum_{k=0}^{n} b_{k} \int_{\Omega} e_{j} \partial_{x}^{4} e_{k},
\end{aligned}
$$

where $X$ is the column vector $\left(b_{0} \ldots b_{n}\right)$ and

$$
F(r, s, t)(X)=\sum_{k, l=0}^{n} b_{k} b_{l}\left(r \int_{\Omega} \partial_{x} e_{k} \partial_{z} e_{l} \partial_{x} e_{j}+s \int_{\Omega} \partial_{x} e_{k} \partial_{x} e_{l} \partial_{z} e_{j}\right)+\sum_{k, l, m=0}^{n} b_{k} b_{l} b_{m} t \int_{\Omega} \partial_{x} e_{k} \partial_{x} e_{l} \partial_{x} e_{m} \partial_{x} e_{j}
$$

In the same way, we obtain the following initial conditions:

$$
a_{j}(0)=a_{j}^{0}
$$

and

$$
b_{j}(0)=b_{j}^{0} .
$$


Hence, by Cauchy-Lipschitz's theorem, we can conclude that there is a unique maximal solution of (33-36), denoted by $v_{n}$ and $h_{n}$ according to the previous definitions, defined on $\left[0, t_{n}\left[\right.\right.$, and which verify $v_{n}(0)=\Pi_{n}\left(v_{0}\right)$ and $h_{n}(0)=\Pi_{n}\left(h_{0}\right)$.

\subsection{A priori estimates}

In this section, we show that $v_{n}$ and $h_{n}$ (built in the previous section) are defined on $\mathbb{R}$ provided the initial conditions are small enough, and are uniformly bounded in $n$. As in Section 3.1, we note $V_{n}$ and $H_{n}$ the mean value with respect to $x$ of the respective functions $v_{n}$ and $h_{n}$.

Proposition 5.1. Let $E>4, F>\max \left(\frac{E}{\pi^{4}}, \frac{E}{\pi^{2}}\right), v_{0}$ in $\mathrm{L}(\Omega), h_{0}$ in $\mathrm{K}(\Omega)$, and $N_{n}(t)$ the following quantity:

$$
N_{n}(t)=\left|v_{n}\right|_{2}^{2}+E\left|h_{n}\right|_{2}^{2}+\left|\partial_{x}^{2} h_{n}\right|_{2}^{2}+\lambda\left|\partial_{z} h_{n}\right|_{2}^{2}+F\left(\left|V_{n}\right|_{2}^{2}+\left|H_{n}\right|_{2}^{2}\right)
$$

Then there exists $X_{0}>0, \lambda_{0}>0$ such that, if the quantity

$$
N_{0}=\left|v_{0}\right|_{2}^{2}+\left|\partial_{x}^{2} h_{0}\right|_{2}^{2}+E\left|h_{0}\right|_{2}^{2}+\lambda_{0}\left|\partial_{z} h_{0}\right|_{2}^{2}+F\left(\left|V_{0}\right|_{2}^{2}+\left|H_{0}\right|_{2}^{2}\right)
$$

is less that $X_{0}$, then for all $n$ in $\mathbb{N}, N_{n}$ is defined on $\mathbb{R}$ and there exists $M>0$ such that, for all $\lambda$ in $\left(0, \lambda_{0}\right)$, for all $t>0$ and for all $n$ in $\mathbb{N}, N_{n}(t) \leqslant M N_{0}$.

This proposition needs two lemmas:

Lemma 5.2. Let $A$ a positive real, $Q$ a 2-valuated polynomial with positive coefficients and $X_{0}$ the first positive root of $f(z)=-A z+Q(z)$. For all $z_{0}$ in $] 0, X_{0}[$, let $z(t)$ be the maximal solution of the Cauchy problem

$$
\left\{\begin{array}{l}
z^{\prime}(t)=f(z(t)) \\
z(0)=z_{0}
\end{array}\right.
$$

Then $z$ is defined on $\mathbb{R}_{+}$, is decreasing and tends to 0 as $t$ tends to $+\infty$.

Lemma 5.3. For all $n$ in $\mathbb{N}, N_{n}(0) \leqslant N_{0}$.

Proof. The norm of $\Pi_{n}$ is equal to 1 . So, $\left|\Pi_{n}\left(v_{0}\right)\right|_{2} \leqslant\left|v_{0}\right|_{2}$ and $\left|\Pi_{n}\left(h_{0}\right)\right|_{2} \leqslant\left|h_{0}\right|_{2}$. As $\left[\Pi_{n}, \partial_{x}^{2}\right]=0,\left|\partial_{x}^{2} \Pi_{n} h_{0}\right|_{2}=$ $\left|\Pi_{n} \partial_{x}^{2} h_{0}\right|_{2} \leqslant\left|\partial_{x}^{2} h_{0}\right|_{2}$

$\left|\partial_{z} \Pi_{n} h_{0}\right|_{2}^{2}=-\int_{\Omega} \Pi_{n} h_{0} \partial_{z}^{2} \Pi_{n} h_{0}$. As $\left[\Pi_{n}, \partial_{z}^{2}\right]=0$ and $\Pi_{n}$ is a self-adjoint projector,

$$
\left|\partial_{z} \Pi_{n} h_{0}\right|_{2}^{2}=-\int_{\Omega} \Pi_{n} h_{0} \partial_{z}^{2} h_{0}=\int_{\Omega} \partial_{z} \Pi_{n} h_{0} \partial_{z} h_{0} \leqslant\left|\partial_{z} \Pi_{n} h_{0}\right|_{2}\left|\partial_{z} h_{0}\right|_{2} .
$$

This concludes the proof.

Proof of Proposition 5.1. Thanks to Section 5.1, $N_{n}(t)$ is defined on $\left[0, t_{n}[\right.$. We apply Theorem 4.1 with $C<\min \left(\frac{1}{2}, \frac{2}{C_{p}}\right)$ and we get $A, \lambda_{0}$ and $Q$, independant of $n$ such that, for all $n$, for all $t$ in $\left[0, t_{n}[\right.$ and for all $\lambda$ in $\left(0, \lambda_{0}\right)$,

$$
\tilde{N}_{n}^{\prime}(t) \leqslant-A \tilde{N}_{n}(t)+Q\left(\tilde{N}_{n}(t)\right)
$$

Let $y=\tilde{N}_{n}$. As $\tilde{N}_{n}$ and $N_{n}$ are equivalent, there exists $M>0$ such that $y(0) \leqslant M N_{n}(0)$. By Lemma 5.3, $y(0) \leqslant M N_{0}$. So, $y$ satisfies:

$$
\left\{\begin{array}{l}
y^{\prime}(t) \leqslant f(y(t)) \\
y(0) \leqslant M N_{0}
\end{array}\right.
$$


Let $z_{0}=M N_{0}$ and $z$ the solution of the differential equation of Lemma 5.2. If we take $X_{0}=\frac{x_{0}}{M}$, where $x_{0}$ is the first positive root of $-A z+Q(z)$, the conclusion of Lemma 5.2 remains. Then classical results on differential equations give $y$ defined on $\mathbb{R}$ and for all $t \in \mathbb{R}, 0 \leqslant y(t) \leqslant z(t)$. As $z$ is decreasing, for all $t \in \mathbb{R}, y(t) \leqslant M N_{0}$. The fact that $N_{n}$ and $\tilde{N}_{n}$ are equivalent finishes the proof.

\subsection{Existence of the limit}

We define here two functions $v$ and $h$ which are the limit of $v_{n}$ and $h_{n}$, thanks to compactness of the weak* topology.

From Proposition 5.1 and from the viscous terms given by (26), we get the following bounds:

$$
\begin{aligned}
& v_{n} \text { is in a bounded set of } \mathrm{L}^{\infty}\left(\mathbb{R}_{+} ; \mathrm{L}(\Omega)\right) \cap \mathrm{L}^{2}\left(\mathbb{R}_{+} ; \mathrm{H}(\Omega)\right), \\
& h_{n} \text { is in a bounded set of } \mathrm{L}^{\infty}\left(\mathbb{R}_{+} ; \mathrm{K}(\Omega)\right) \cap \mathrm{L}^{2}\left(\mathbb{R}_{+} ; \mathrm{H}^{2}(\Omega) \cap \mathrm{H}(\Omega)\right), \\
& \partial_{x}^{2} \partial_{z} h_{n} \text { is in a bounded set of } \mathrm{L}^{2}\left(\mathbb{R}_{+} \times \Omega\right), \\
& \partial_{x}^{4} h_{n} \text { is in a bounded set of } \mathrm{L}^{2}\left(\mathbb{R}_{+} \times \Omega\right) .
\end{aligned}
$$

Then, up to an extraction of a subsequence, inclusions (37-40) imply that there exists

$$
v \in \mathrm{L}^{\infty}\left(\mathbb{R}_{+} ; \mathrm{L}(\Omega)\right) \cap \mathrm{L}^{2}\left(\mathbb{R}_{+} ; \mathrm{H}(\Omega)\right)
$$

and

$$
h \in \mathrm{L}^{\infty}\left(\mathbb{R}_{+} ; \mathrm{K}(\Omega)\right) \cap \mathrm{L}^{2}\left(\mathbb{R}_{+} ; \mathrm{H}^{2}(\Omega) \cap \mathrm{H}(\Omega)\right)
$$

such that $\partial_{x}^{2} \partial_{z} h \in \mathrm{L}^{2}\left(\mathbb{R}_{+} \times \Omega\right)$ and $\partial_{x}^{4} h \in \mathrm{L}^{2}\left(\mathbb{R}_{+} \times \Omega\right)$ and

$$
\begin{aligned}
v_{n} \rightarrow & v \text { weak } * \text { in } \mathrm{L}^{\infty}\left(\mathbb{R}_{+} ; \mathrm{L}(\Omega)\right) \text { and weakly in } \mathrm{L}^{2}\left(\mathbb{R}_{+} ; \mathrm{H}(\Omega)\right), \\
h_{n} \rightarrow & h \text { weak } * \text { in } \mathrm{L}^{\infty}\left(\mathbb{R}_{+} ; \mathrm{K}(\Omega)\right) \text { and } \\
& \text { weakly in } \mathrm{L}^{2}\left(\mathbb{R}_{+} ; \mathrm{H}^{2}(\Omega) \cap \mathrm{H}(\Omega)\right), \\
\partial_{x}^{2} \partial_{z} h_{n} \rightarrow & \partial_{x}^{2} \partial_{z} h \text { weakly in } \mathrm{L}^{2}\left(\mathbb{R}_{+} \times \Omega\right), \\
\partial_{x}^{4} h_{n} \rightarrow & \partial_{x}^{4} h \text { weakly in } \mathrm{L}^{2}\left(\mathbb{R}_{+} \times \Omega\right) .
\end{aligned}
$$

To conclude that $v_{n}$ and $h_{n}$ verify (3-4), we will use compactness in $\mathrm{L}^{2}(0, \mathrm{~T} ; \mathrm{X})$, that is to say:

Proposition 5.4. Up to an extraction of a subsequence, for $T>0$,

$$
\begin{aligned}
v_{n} & \rightarrow v \\
h_{n} & \rightarrow h \\
\partial_{x} h_{n} & \rightarrow \partial_{x} h \\
\partial_{z} h_{n} & \rightarrow \partial_{z} h
\end{aligned}
$$

strong in $\mathrm{L}^{2}((0, \mathrm{~T}) \times \Omega)$ and a.e.

This proposition requires the fact that $\partial_{t} v_{n}, \partial_{t} h_{n}, \partial_{t} \partial_{x} h_{n}$ and $\partial_{t} \partial_{z} h_{n}$ are bounded in $\mathrm{L}^{2}\left(0, \mathrm{~T} ; \mathrm{H}(\Omega)^{\prime}\right)$ and needs the following lemma:

Lemma 5.5. Let $\mathrm{H}_{1} \hookrightarrow \mathrm{H} \hookrightarrow \mathrm{H}_{2}$ three hilbert spaces so that the first embedding is compact. Then for any $p_{1}$ and $p_{2}$ in $(1,+\infty)$, the embedding

$$
\left\{f \in \mathrm{L}^{p_{1}}\left(0, T ; \mathrm{H}_{1}\right), \frac{\mathrm{d} f}{\mathrm{~d} t} \in \mathrm{L}^{p_{2}}\left(0, T ; \mathrm{H}_{2}\right)\right\} \hookrightarrow \mathrm{L}^{p_{1}}(0, T ; \mathrm{H})
$$

is compact. 
For a proof, see [10] (p. 58).

Hence, nonlinear terms must lay in a bounded set of $\mathrm{L}^{2}((0, T) \times \Omega)$ :

Lemma 5.6. The nonlinear terms in equations (29) and (30) verified by $v_{n}$ and $h_{n}$ are in a bounded set of $\mathrm{L}^{2}((0, T) \times \Omega)$.

Proof. For sake of simplicity, we note $h$ instead of $h_{n}$. As $h$ is periodic in the direction $x$, integrating by parts with respect to $x$ does not add any boundary terms. We first show three inequalities we will use throughout this proof.

We recall that $h$ verifies (38-40). This fact and Lemma 4.4 show that there exists $M>0$ such that, for all $p \in[2,6]$ and for all $t \in[0, T]$,

$$
\left|\partial_{x} h\right|_{p} \leqslant M
$$

In the same way, Lemma 4.3 implies that, for $p \geqslant 1$, there exists $M>0$ such that, for all $t \in[0, T]$,

$$
|h|_{p}^{p} \leqslant M|h|_{p-2}^{p-2}\left(\left|\partial_{x} h\right|_{2}^{2}+\left|\partial_{z} h\right|_{2}^{2}\right) \leqslant M|h|_{p-2}^{p-2},
$$

and that there exists $M>0$ such that

$$
\left|\partial_{z} h\right|_{4}^{\frac{15}{4}} \leqslant M\left(\left|\partial_{z} h\right|_{2}^{30}+\left|\partial_{x}^{2} h\right|_{2}^{2}+\left|\partial_{z}^{2} h\right|_{2}^{2}\right) .
$$

- First, let show the result on $\partial_{z}\left(\left(\partial_{x} h\right)^{2}\right)$ :

As $\partial_{z}\left(\left(\partial_{x} h\right)^{2}\right)=2 \partial_{x} \partial_{z} h \partial_{x} h$, let

$$
I=\int_{\Omega}\left(\partial_{x} \partial_{z} h\right)^{2}\left(\partial_{x} h\right)^{2}=-2 \int_{\Omega} \partial_{z} h \partial_{x} \partial_{z} h \partial_{x}^{2} h \partial_{x} h-2 \int_{\Omega} \partial_{z} h \partial_{x}^{2} \partial_{z} h\left(\partial_{x} h\right)^{2},
$$

by integrating by parts with respect to $x$. Let $-2 I_{1}$ be equal to the first integral and $-2 I_{2}$ to the second. It is sufficient to show that $I_{1}$ and $I_{2}$ are in a bounded set of $\mathrm{L}^{1}(0, T)$.

By integrating $I_{1}$ by parts with respect to $x$, we get $I_{1}=-I_{1}-I_{3}-I_{4}$ where $I_{3}=\int_{\Omega}\left(\partial_{z} h\right)^{2} \partial_{x}^{3} h \partial_{x} h$ and $I_{4}=\int_{\Omega}\left(\partial_{z} h\right)^{2}\left(\partial_{x}^{2} h\right)^{2}$. Hence

$I_{3} \leqslant\left|\partial_{z} h\right|_{4}^{2}\left|\partial_{x} h\right|_{4}\left|\partial_{x}^{3} h\right|_{4}$, and the proof of Proposition 4.9 shows that $\left|\partial_{x}^{3} h\right|_{4} \leqslant M\left|\partial_{x}^{2} h\right|_{2}^{\frac{1}{8}}\left|\partial_{x}^{4} h\right|_{2}^{\frac{1}{8}}\left|\partial_{x}^{3} h\right|_{6}^{\frac{3}{4}}$.

$$
\begin{aligned}
I_{3} & \leqslant M\left|\partial_{z} h\right|_{4}^{2}\left|\partial_{x} h\right|_{4}\left|\partial_{x}^{2} h\right|_{2}^{\frac{1}{8}}\left|\partial_{x}^{4} h\right|_{2}^{\frac{1}{8}}\left|\partial_{x}^{3} h\right|_{6}^{\frac{3}{4}} \\
& \leqslant M\left(\left|\partial_{z} h\right|_{4}^{\frac{15}{4}}+\left|\partial_{x}^{4} h\right|_{2}^{2}+\left|\partial_{x} h\right|_{4}^{60}+\left|\partial_{x}^{2} h\right|_{2}^{80}+\left|\partial_{x}^{3} h\right|_{6}^{2}\right) \\
& \leqslant M\left(\left|\partial_{z} h\right|_{4}^{\frac{15}{4}}+\left|\partial_{x}^{4} h\right|_{2}^{2}+\left|\partial_{x} h\right|_{4}^{60}+\left|\partial_{x}^{2} h\right|_{2}^{80}+\left|\partial_{x}^{2} \partial_{z} h\right|_{2}^{2}+\left|\partial_{x}^{4} h\right|_{2}^{2}\right)
\end{aligned}
$$

by using Lemma 4.4 for the last term of the second inequality. According to $(38-40,49,51), I_{3}$ is in a bounded set of $\mathrm{L}^{1}(0, T)$ (we recall that $h$ replaces $h_{n}$ ).

Now, let us show that $I_{4}$ is in a bounded set of $\mathrm{L}^{1}(0, T)$ to conclude that $I_{1}$ verifies the same property:

$$
I_{4} \leqslant\left|\partial_{z} h\right|_{4}^{2}\left|\partial_{x}^{2} h\right|_{4}^{2} \leqslant M\left(\left|\partial_{z} h\right|_{4}^{\frac{15}{4}}+\left|\partial_{x}^{2} h\right|_{4}^{\frac{30}{7}}\right) .
$$


Using Proposition 4.9 and the fact that $\left|\partial_{x}^{2} h\right|_{2}$ is in a bounded set of $\mathrm{L}^{\infty}(0, T)(c f .(38))$ leads to

$$
\begin{aligned}
I_{4} & \leqslant M\left(\left|\partial_{z} h\right|_{4}^{\frac{15}{4}}+\left|\partial_{x} h\right|_{4}^{\frac{240}{7}}+\left|\partial_{x}^{4} h\right|_{2}^{2}+\left|\partial_{x}^{3} h\right|_{6}^{2}\right) \\
& \leqslant M\left(\left|\partial_{z} h\right|_{4}^{\frac{15}{4}}+\left|\partial_{x} h\right|_{4}^{\frac{240}{7}}\left|\partial_{x}^{2} \partial_{z} h\right|_{2}^{2}+\left|\partial_{x}^{4} h\right|_{2}^{2}\right)
\end{aligned}
$$

by using Lemma 4.4 for the last term of the first inequality. Hence it is easy to conclude that $I_{4}$ is in a bounded set of $\mathrm{L}^{1}(0, T)$ with $(39,40,49,51)$, so does $I_{1}$.

Finally,

$$
I_{2} \leqslant\left|\partial_{x}^{2} \partial_{z} h\right|_{2}\left|\partial_{z} h\right|_{4}\left|\partial_{x} h\right|_{8}^{2}
$$

Using Lemma 4.3 leads to

$$
I_{2} \leqslant M\left|\partial_{x}^{2} \partial_{z} h\right|_{2}\left(\left|\partial_{x}^{2} h\right|_{2}^{2}+\left|\partial_{z}^{2} h\right|_{2}^{2}\right)^{\frac{1}{2}}\left|\partial_{z} h\right|_{2}^{\frac{1}{2}}\left|\partial_{x} h\right|_{6}^{\frac{3}{2}}
$$

Using (49) and (38) leads to

$$
I_{2} \leqslant M\left(\left|\partial_{x}^{2} \partial_{z} h\right|_{2}^{2}+\left|\partial_{x}^{2} h\right|_{2}^{2}+\left|\partial_{z}^{2} h\right|_{2}^{2}\right)
$$

which is in a bounded set of $\mathrm{L}^{1}(0, T)$ thanks to (38) and (39).

- Now we deal with $\partial_{x}\left(\partial_{x} h \partial_{z} h\right)$ which is equal to $\partial_{x}^{2} h \partial_{z} h+\partial_{x} \partial_{z} h \partial_{x} h$. The last term is the one treated previously and showing the result on the first one is dealing with $I_{4}$. Hence $\partial_{x}\left(\partial_{x} h \partial_{z} h\right)$ is in a bounded set of $\mathrm{L}^{2}((0, T) \times \Omega)$.

- Finally, $\partial_{x}\left(\left(\partial_{x} h\right)^{3}\right)=3 \partial_{x}^{2} h\left(\partial_{x} h\right)^{2}$. Let $I=\int_{\Omega}\left(\partial_{x}^{2} h\right)^{2}\left(\partial_{x} h\right)^{4}$. By integrating by parts with respect to $x$, one has $I=-3 I_{5}-2 I_{6}$, where $I_{5}=\int_{\Omega} h\left(\partial_{x}^{2} h\right)^{3}\left(\partial_{x} h\right)^{2}$ and $I_{6}=\int_{\Omega} h \partial_{x}^{3} h \partial_{x}^{2} h\left(\partial_{x} h\right)^{3}$. Using Proposition 4.9 leads to

$$
\begin{aligned}
I_{5} & \leqslant|h|_{12}\left|\partial_{x} h\right|_{6}^{2}\left|\partial_{x}^{2} h\right|_{4}^{3} \\
& \leqslant|h|_{12}\left|\partial_{x} h\right|_{6}^{2}\left|\partial_{x} h\right|_{4}^{\frac{3}{2}}\left|\partial_{x}^{2} h\right|_{2}^{\frac{3}{16}}\left|\partial_{x}^{4} h\right|_{2}^{\frac{3}{16}}\left|\partial_{x}^{3} h\right|_{6}^{\frac{9}{8}}
\end{aligned}
$$

Using (49) and (50) leads to

$$
\begin{aligned}
I_{5} & \leqslant M|h|_{2}^{\frac{2}{3}}\left|\partial_{x} h\right|_{4}^{\frac{3}{2}}\left|\partial_{x}^{2} h\right|_{2}^{\frac{3}{16}}\left|\partial_{x}^{4} h\right|_{2}^{\frac{3}{16}}\left|\partial_{x}^{3} h\right|_{6}^{\frac{9}{8}} \\
& \leqslant M\left(\left|\partial_{x}^{2} h\right|_{2}^{2}+\left|\partial_{x}^{4} h\right|_{2}^{2}+\left|\partial_{x}^{3} h\right|_{6}^{2}\right) \\
& \leqslant M\left(\left|\partial_{x}^{2} h\right|_{2}^{2}+\left|\partial_{x}^{2} \partial_{z} h\right|_{2}^{2}+\left|\partial_{x}^{4} h\right|_{2}^{2}\right)
\end{aligned}
$$

according to Lemma 4.4. Then, it is easy to conclude that $I_{5}$ is in a bounded set of $\mathrm{L}^{1}(0, T)$ thanks to $(38-40)$.

Now, we deal with $I_{6}$ which is equal to $-I_{6}-I_{7}-I_{8}-2 I_{9}$ by integrating by parts with respect to $x$, where $I_{7}=\int_{\Omega} h^{2} \partial_{x}^{4} h \partial_{x}^{2} h\left(\partial_{x} h\right)^{2}, I_{8}=\int_{\Omega} h^{2}\left(\partial_{x}^{3} h\right)^{2}\left(\partial_{x} h\right)^{2}$ and $I_{9}=\int_{\Omega} h^{2} \partial_{x} h\left(\partial_{x}^{2} h\right)^{2} \partial_{x}^{3} h$. Then

$$
\begin{aligned}
I_{7} & \leqslant|h|_{15}^{2}\left|\partial_{x}^{4} h\right|_{2}\left|\partial_{x} h\right|_{6}^{2}\left|\partial_{x}^{2} h\right|_{5} \\
& \leqslant|h|_{15}^{2}\left|\partial_{x}^{4} h\right|_{2}\left|\partial_{x} h\right|_{6}^{2}\left|\partial_{x}^{2} h\right|_{2}^{\frac{1}{10}}\left|\partial_{x}^{2} h\right|_{6}^{\frac{9}{10}}
\end{aligned}
$$


by using classical results on interpolation. As $|h|_{15}$ is in a bounded set of $\mathrm{L}^{\infty}(0, T)$ (thanks to (50) and (38)) as well as $\left|\partial_{x} h\right|_{6}$ (thanks to (49) and (38)), $I_{7} \leqslant M\left|\partial_{x}^{4} h\right|_{2}\left|\partial_{x}^{2} h\right|_{2}^{\frac{1}{10}}\left|\partial_{x}^{3} h\right|_{6}^{\frac{9}{10}} \leqslant M\left(\left|\partial_{x}^{4} h\right|_{2}^{2}+\left|\partial_{x}^{2} h\right|_{2}^{2}+\left|\partial_{x}^{3} h\right|_{6}^{2}\right)$. To conclude that $I_{7}$ is in a bounded set of $\mathrm{L}^{1}(0, T)$, it suffices to apply Lemma 4.4 to the last term of this inequality and to conclude with (38) and (39).

Now we estimate $I_{8}$ which is less or equal than $|h|_{12}^{2}\left|\partial_{x} h\right|_{6}^{2}\left|\partial_{x}^{3} h\right|_{4}^{2}$. We have already seen that $\left|\partial_{x}^{3} h\right|_{4} \leqslant$ $M\left|\partial_{x}^{2} h\right|_{2}^{\frac{1}{8}}\left|\partial_{x}^{4} h\right|_{2}^{\frac{1}{8}}\left|\partial_{x}^{3} h\right|_{6}^{\frac{3}{4}}$. Then

$$
I_{8} \leqslant M|h|_{12}^{2}\left|\partial_{x} h\right|_{6}^{2}\left|\partial_{x}^{2} h\right|_{2}^{\frac{1}{4}}\left|\partial_{x}^{4} h\right|_{2}^{\frac{1}{4}}\left|\partial_{x}^{3} h\right|_{6}^{\frac{3}{2}}
$$

As usual, $|h|_{12}^{2}\left|\partial_{x} h\right|_{6}^{2}$ is in a bounded set of $L^{\infty}(0, T)$. Then

$$
I_{8} \leqslant M\left|\partial_{x}^{2} h\right|_{2}^{\frac{1}{4}}\left|\partial_{x}^{4} h\right|_{2}^{\frac{1}{4}}\left|\partial_{x}^{3} h\right|_{6}^{\frac{3}{2}} \leqslant M\left(\left|\partial_{x}^{2} h\right|_{2}^{2}+\left|\partial_{x}^{4} h\right|_{2}^{2}+\left|\partial_{x}^{3} h\right|_{6}^{2}\right)
$$

We conclude as for $I_{7}$.

It remains $I_{9}$ which is less or equal than $|h|_{24}^{2}\left|\partial_{x} h\right|_{6}\left|\partial_{x}^{3} h\right|_{4}\left|\partial_{x}^{2} h\right|_{4}^{2}$. By using Proposition 4.9 and the bound of $\left|\partial_{x}^{3} h\right|_{4}$, we get

$$
I_{9} \leqslant M|h|_{24}^{2}\left|\partial_{x} h\right|_{6}\left|\partial_{x} h\right|_{4}\left|\partial_{x}^{2} h\right|_{2}^{\frac{1}{4}}\left|\partial_{x}^{4} h\right|_{2}^{\frac{1}{4}}\left|\partial_{x}^{3} h\right|_{6}^{\frac{3}{2}}
$$

As usual, $|h|_{24}^{2}\left|\partial_{x} h\right|_{6}\left|\partial_{x} h\right|_{4}$ is in a bounded set of $\mathrm{L}^{\infty}(0, T)$ and $I_{9} \leqslant M\left(\left|\partial_{x}^{2} h\right|_{2}^{2}+\left|\partial_{x}^{4} h\right|_{2}^{2}+\left|\partial_{x}^{3} h\right|_{6}^{2}\right)$. We conclude as for $I_{7}$ and the proof is finished.

Proof of Proposition 5.4. From Lemma 5.5 (with $p_{1}=p_{2}=2, \mathrm{H}_{1}=\mathrm{H}(\Omega), \mathrm{H}_{2}=\mathrm{H}(\Omega)^{\prime}$, H $=\mathrm{L}^{2}(\Omega)$ ), it's easy to see that we only need that

- For all $T>0, \partial_{t} v_{n}$ and $\partial_{t} h_{n}$ are bounded in $\mathrm{L}^{2}\left(0, T ; \mathrm{H}(\Omega)^{\prime}\right)$ :

Let us fix $T>0$. (37) implies that $v_{n}, \partial_{x}^{2} v_{n}$ and $\partial_{z}^{2} v_{n}$ are in a bounded set of $\mathrm{L}^{2}\left((0, T), \mathrm{H}(\Omega)^{\prime}\right)$.

As $z$ lies in $(0,1)$, the same conclusion remains for $\frac{z}{\lambda} \partial_{x} v_{n}$.

Thanks to (38) and (40), $\frac{z}{\lambda} \partial_{x} h_{n}, \partial_{x}^{2} h_{n}, \partial_{z}^{2} h_{n}$ and $\partial_{x}^{4} h_{n}$ remain in a bounded set of $\mathrm{L}^{2}((0, T) \times \Omega)$ which is continuously embedded in $\mathrm{L}^{2}\left((0, T) ; \mathrm{H}(\Omega)^{\prime}\right)$.

It only remains the nonlinear terms. Lemma 5.6 shows that they are in a bounded set of $\mathrm{L}^{2}((0, T) \times \Omega)$, therefore in a bounded set of $\mathrm{L}^{2}\left((0, T) ; \mathrm{H}(\Omega)^{\prime}\right)$.

Then, up to an extraction of a subsequence, for all $T>0, v_{n} \rightarrow v$ strong in $\mathrm{L}^{2}((0, T) \times \Omega)$ and a.e. Obviously, the same result holds on $h$. So (45) and (46) are proved.

- For all $T$ positive real, $\partial_{t} \partial_{x} h_{n}$ and $\partial_{t} \partial_{z} h_{n}$ are in a bounded set of $\mathrm{L}^{2}\left(0, T ; \mathrm{H}(\Omega)^{\prime}\right)$ :

Lemma 5.6 shows that they are in a bounded set of $\mathrm{L}^{2}((0, T) \times \Omega)$, therefore their derivatives with respect to $x$ and $z$ are in a bounded set of $\mathrm{L}^{2}\left((0, T) ; \mathrm{H}(\Omega)^{\prime}\right)$.

So, as for $v_{n}$, we conclude that, for all $T>0, \partial_{x} h_{n} \rightarrow \partial_{x} h$ and $\partial_{z} h_{n} \rightarrow \partial_{z} h$ strong in $\mathrm{L}^{2}((0, T) \times \Omega)$ and a.e., and (47) and (48) are proved.

Remark 5.7. $v$ and $h$ depend a priori on $T$. With a diagonal extraction, we eliminate this fact (up to an extraction of a subsequence...). 


\subsection{Conclusion of the proof of Theorem $\mathbf{2 . 2}$}

We first show that $\partial_{t} v_{n}$ tends to $\partial_{t} v$ so that equation (3) holds:

Proposition 5.8. Let $T>0$. Then, $\partial_{t} v_{n}$ tends towards $\partial_{t} v$ in $\mathrm{L}^{2}\left(0, T ; \mathrm{H}(\Omega)^{\prime}\right)$ and $v$ and $h$ satisfy the equation (3). In addition, $v \in \mathrm{C}^{0}\left([0, T] ; \mathrm{L}^{2}(\Omega)\right)$.

Proof. Let $N \in \mathbb{N}, \Phi:[0, T] \times \Omega \rightarrow \mathbb{R}, \mathrm{C}^{\infty}$ with respect to time, so that $\Phi(T)=\Phi(0)=0$ and, for all $t \in[0, T]$, $\Phi(t,.) \in E_{N}$. Here, (.,.) will denote the inner product on $\mathrm{L}^{2}(\Omega)$ and $n$ will be an integer greater than $N$. The linear terms of $\int_{0}^{T}\left(\partial_{t} v_{n}, \Phi\right)$ do not hold any problem (we use $(41-44,45)$ and Lebesgue's theorem). Let us focus on the nonlinear terms of $\int_{0}^{T}\left(\partial_{t} v_{n}, \Phi\right)$, which are $\int_{0}^{T} \int_{\Omega} \partial_{x}\left(\partial_{x} h_{n} \partial_{z} h_{n}\right) \Phi \mathrm{d} t, \int_{0}^{T} \int_{\Omega} \partial_{z}\left(\left(\partial_{x} h_{n}\right)^{2}\right) \Phi \mathrm{d} t$ and $\int_{0}^{T} \int_{\Omega} \partial_{x}\left(\left(\partial_{x} h_{n}\right)^{3}\right) \Phi \mathrm{d} t$. Thanks to $(47,48)$ and Lebesgue's theorem, we have:

$$
\begin{gathered}
\int_{0}^{T} \int_{\Omega} \partial_{x} h_{n} \partial_{z} h_{n} \partial_{x} \Phi \mathrm{d} t \underset{n \rightarrow \infty}{\longrightarrow} \int_{0}^{T} \int_{\Omega} \partial_{x} h \partial_{z} h \partial_{x} \Phi \mathrm{d} t \\
\int_{0}^{T} \int_{\Omega}\left(\partial_{x} h_{n}\right)^{2} \partial_{z} \Phi \mathrm{d} t \underset{n \rightarrow \infty}{\longrightarrow} \int_{0}^{T} \int_{\Omega}\left(\partial_{x} h\right)^{2} \partial_{z} \Phi \mathrm{d} t
\end{gathered}
$$

and

$$
\int_{0}^{T} \int_{\Omega}\left(\partial_{x} h_{n}\right)^{3} \partial_{x} \Phi \mathrm{d} t \underset{n \rightarrow \infty}{\longrightarrow} \int_{0}^{T} \int_{\Omega}\left(\partial_{x} h\right)^{3} \partial_{x} \Phi \mathrm{d} t .
$$

As, $\left(\partial_{t} v_{n}, \Phi\right)=<\partial_{t} v_{n}, \Phi>_{\mathrm{H}(\Omega)^{\prime}, \mathrm{H}(\Omega)}$ and

$$
\int_{0}^{T}\left(\partial_{t} v_{n}, \Phi\right) \mathrm{d} t=-\int_{0}^{T}\left(v_{n}, \partial_{t} \Phi\right) \mathrm{d} t \underset{n \rightarrow \infty}{\longrightarrow}-\int_{0}^{T}\left(v, \partial_{t} \Phi\right) \mathrm{d} t
$$

We have perform the limit for each $\Phi$ in $E_{N}$ and for all $N$, this means that $v$ and $h$ verify the equation (3) in a weak sense, in $\mathrm{L}^{2}\left(0, T ; \mathrm{H}(\Omega)^{\prime}\right)$.

Finally, Lemma 3.10 involves $v \in \mathrm{C}^{0}\left([0, T] ; \mathrm{L}^{2}(\Omega)\right)$, which concludes the proof of the proposition.

The initial conditions are treated in the usual way and we have $v(0)=v_{0}$.

The same result holds for equation (4):

Proposition 5.9. Let $T>0$. Then, $\partial_{t} h_{n}$ tends to $\partial_{t} h$ in $\mathrm{L}^{2}\left(0, T ; \mathrm{H}(\Omega)^{\prime}\right)$ and $v$ and $h$ verify the equation (4). In addition, $h \in \mathrm{C}^{0}\left([0, T] ; \mathrm{L}^{2}(\Omega)\right)$.

Proof. We choose $N$ and $\Phi$ as in Proposition 5.8. The linear terms do not hold any problem: we use (45) and Lebesgue's theorem. Then, $v$ and $h$ verify the equation (4) in a weak sense. We apply Lemma 3.10 to conclude that $h \in \mathrm{C}^{0}\left([0, T] ; \mathrm{L}^{2}(\Omega)\right)$, hence the proof is finished.

Finally, the initial condition of $h$ is treated as for $v$ and we have $h(0)=h_{0}$. This concludes the proof of Theorem 2.2.

\section{Proof of the LOCAL EXISTENCE}

We will use exactly the same arguments as for the global existence.

\subsection{Approximation of the solution}

As in Section 5.1, we find $v_{n}$ and $h_{n}$ in $\mathrm{C}^{\infty}\left(\left[0, t_{n}\right) ; E_{n}\right)$, unique maximal solution of $(29)$ and (30), verifying $v_{n}(0)=\Pi_{n}\left(v_{0}\right)$ and $h_{n}(0)=\Pi_{n}\left(h_{0}\right)$. Of course, $v_{n}$ and $h_{n}$ are 2-periodic in $x$ and vanish at $z=0,1$. 


\subsection{A priori estimates}

We denote $N_{n}(t)$ the quantity $\left|v_{n}\right|_{2}^{2}+\epsilon_{1} k^{4}\left|h_{n}\right|_{2}^{2}+\left|h_{n}\right|_{2}^{2}$. As for global existence, this section shows that $N_{n}$ is uniformly bounded in $n$ :

Proposition 6.1. Let $v_{0}$ in $\mathrm{L}(\Omega), h_{0}$, in $\mathrm{K}(\Omega)$. There exists $T_{e}>0$ such that, for all $T$ in $\left[0, T_{e}\right)$, there exists $M>0$ such that, for all $n$ in $\mathbb{N}$, for all $t$ in $[0, T], N_{n}(t) \leqslant N_{o} M$.

Proof. We make the same computations as in Theorem 4.2 and this gives $Q$ (1-valuated polynomial with positive coefficients) such that $N_{n}^{\prime}(t) \leqslant Q\left(N_{n}(t)\right)$, whose time existence is $t_{n}$. As for global existence, let us consider the maximal solution $z$ of the differential equation

$$
\left\{\begin{array}{l}
z^{\prime}(t)=Q(z(t)) \\
z(0)=\left|v_{0}\right|_{2} 2+\epsilon_{1}\left|\partial_{x}^{2} h_{0}\right|_{2}^{2}+\left|\partial_{z} h_{0}\right|_{2}^{2},
\end{array}\right.
$$

whose time definition is denoted $T_{e}$. By classical results on differential equations, $t_{n} \geqslant T_{e}$ and $N_{n}(t) \leqslant z(t)$, for all $t$ in $\left[0, T_{e}[\right.$. Let $T \in] 0, T_{e}[, \tilde{Q}$ such that $Q(X)=X \tilde{Q}(X)$ (possible since $Q$ is a 1-valuated polynomial), $M_{1}(T)=\sup _{[0, T]} z(t), M(T)=\tilde{Q}\left(M_{1}(T)\right)$ and $M=\exp (T M(T))$. Since $N_{n}(t) \leqslant z(t)$, we have

$$
N_{n}^{\prime}(t) \leqslant N_{n} \tilde{Q}\left(N_{n}\right) \leqslant N_{n} \tilde{Q}(z) \leqslant y=N_{n} \tilde{Q}\left(M_{1}(T)\right) \leqslant N_{n} M(T) .
$$

By Gronwall's lemma, for all $t$ in $[0, T]$,

$$
N_{n}(t) \leqslant N_{n}(0) \exp (T M(T)) \leqslant z(0) \exp (T M(T)) .
$$

The same arguments as in Theorem 5.3 give $N_{n}(0) \leqslant N_{0}=z(0)$. So

$$
\sup _{t \in[0, T]}\left(N_{n}(t)\right) \leqslant N_{0} M .
$$

This result and the viscous terms given by the Theorem 4.2 imply that, for all $T<T_{e}$,

$$
\begin{aligned}
& v_{n} \text { is in a bounded set of } \mathrm{L}^{\infty}(0, T ; \mathrm{L}(\Omega)) \cap \mathrm{L}^{2}(0, T ; \mathrm{H}(\Omega)), \\
& h_{n} \text { is in a bounded set of } \mathrm{L}^{\infty}(0, T ; \mathrm{K}(\Omega)) \cap \mathrm{L}^{2}\left(0, T ; \mathrm{H}^{2}(\Omega) \cap \mathrm{H}(\Omega)\right), \\
& \partial_{x}^{2} \partial_{z} h_{n} \text { is in a bounded set of } \mathrm{L}^{2}((0, T) \times \Omega), \\
& \partial_{x}^{4} h_{n} \text { is in a bounded set of } \mathrm{L}^{2}((0, T) \times \Omega) .
\end{aligned}
$$

\subsection{Existence of the limit}

Up to an extraction of a subsequence, equations (52-55) imply that there exists

$$
v \text { in } \mathrm{L}^{\infty}(0, T ; \mathrm{L}(\Omega)) \cap \mathrm{L}^{2}(0, T ; \mathrm{H}(\Omega))
$$

and

$$
h \text { in } \mathrm{L}^{\infty}(0, T ; \mathrm{K}(\Omega)) \cap \mathrm{L}^{2}\left(0, T ; \mathrm{H}^{2}(\Omega) \cap \mathrm{H}(\Omega)\right)
$$


such that $\partial_{x}^{2} \partial_{z} h \in \mathrm{L}^{2}((0, T) \times \Omega)$ and $\partial_{x}^{4} h \in \mathrm{L}^{2}((0, T) \times \Omega)$ and

$$
\begin{aligned}
v_{n} \rightarrow & v \text { weak } * \text { in } \mathrm{L}^{\infty}(0, T ; \mathrm{L}(\Omega)) \text { and weakly in } \mathrm{L}^{2}(0, T ; \mathrm{H}(\Omega)), \\
h_{n} \rightarrow & h \text { weak } * \text { in } \mathrm{L}^{\infty}(0, T ; \mathrm{K}(\Omega)) \text { and } \\
& \text { weakly in } \mathrm{L}^{2}\left(0, T ; \mathrm{H}^{2}(\Omega) \cap \mathrm{H}(\Omega)\right), \\
\partial_{x}^{2} \partial_{z} h_{n} \rightarrow & \partial_{x}^{2} \partial_{z} h \text { weakly in } \mathrm{L}^{2}((0, T) \times \Omega), \\
\partial_{x}^{4} h_{n} \rightarrow & \partial_{x}^{4} h \text { weakly in } \mathrm{L}^{2}((0, T) \times \Omega) .
\end{aligned}
$$

As for the global existence, $v$ and $h$ will verify the equations (3) and (4) by using compactness in the time spaces (Arguments of Prop. 5.4 remain). Hence we conclude that (45-47) and (48) hold.

Remark 6.2. Of course it is possible to find $v$ and $h$ independant of $T$ by using a diagonal extraction.

\subsection{Conclusion}

The arguments given in the proof of the global existence are valid but instead of using (41-44), we use $(56-59)$. The case of the initial condition $(t=0)$ is proved in the same way. So the proof of Theorem 2.4 is completed.

\section{Conclusion}

The mathematical results exposed in this paper explain the stabilization of lamellar phases observed in the experiments (Th. 2.2): instabilities does not develop. More over, the linear system is controlled by a nonconstant parameter and is stable at high shear, i.e. when this parameter is high (Th. 2.1). It shows that small scales are always stable and that the linear system tends to its rest state.

The author would like to thank A. Colin and D. Roux for their physical discussions on the model and especially Professor T. Colin for his patience and his interest for this work.

\section{REFERENCES}

[1] O.V. Besov, V.P. Il'in and S.M. Nikol'skii, Integral representations of functions and embeddings theorems, Vol. 1. V.H. Winston and Sons.

[2] A. Babin, B. Nicolaenko and A. Mahalov, Regularity and integrability of $3 D$ Euler and Navier-Stokes equations for rotating fluids. Asymptot. Anal. 15 (1997) 103-150.

[3] A. Colin, T. Colin, D. Roux and A.S. Wunenburger, Undulation instability under shear: A model to explain the different orientation of a lamellar phase under shear. European J. Soft Condensed Matter (to appear).

[4] A. de Bouard and J.C. Saut, Solitary waves of generalized Kadomtsev-Petviashvili equations. Ann. Inst. H. Poincaré Anal. Non Linéaire 14 (1997) 211-236.

[5] O. Diat, D. Roux and F. Nallet. J. Phys. II France 3 (1993) 1427.

[6] O. Diat and D. Roux. J. Phys. II France 3 (1993) 9.

[7] I. Gallagher, Asymptotic of solutions of hyperbolic equations with a skew-symmetric perturbation. J. Differential Equations 150 (1998) 363-384.

[8] E. Grenier, Oscillatory perturbations of the Navier-Stokes equations. J. Math. Pures Appl. 76 (1997) 477-498.

[9] O.A. Ladyzhenskaya, The Mathematical Theory of Viscous Incompressible Flow. Gordon and Breach (1963).

[10] J.L. Lions, Quelques méthodes de résolution des problèmes aux limites non linéaires. Dunod (1969).

[11] J.L. Lions and E. Magenes, Problèmes aux limites non homogènes et applications. Dunod (1968).

[12] J. Simon, Compact sets in the Spaces $L^{p}(0, T ; B)$. Ann. Mat. Pura Appl. 146 (1987) 65-96.

[13] R. Temam, Infinite-dimensional dynamical systems in mechanics and physics. Springer-Verlag, Appl. Math. Sci. 68 (1997). 